\title{
Flow Boiling and Frictional Pressure Gradients in Plate Heat Exchangers: Part 2, Comparison of Literature Methods to Database and New Prediction Methods
}

\author{
Raffaele L. Amalfi ${ }^{\text {a }}$, Farzad Vakili-Farahani ${ }^{b}$, John R. Thome ${ }^{\mathrm{c}}$, john.thome@epfl.ch \\ Laboratory of Heat and Mass Transfer (LTCM), École Polytechnique Fédérale de Lausanne (EPFL) \\ EPFL-STI-IGM-LTCM, Station 9, CH-1015, Lausanne, Switzerland a,c \\ Swiss Federal Laboratories for Materials Science and Technology (Empa) \\ Feuerwerkerstrasse 39, CH-3602 Thun, Bern, Switzerland ${ }^{\text {b }}$ \\ Corresponding author \\ Raffaele L. Amalfi \\ Tel: +41-21-693-54-42 \\ E-mail: raffaelelucaamalfi@gmail.com, luca.amalfi@epfl.ch
}

\begin{abstract}
In the second part of this study a sensitivity analysis on the prediction methods is performed to consider the effect of plate geometry on thermal-hydraulic performance and an extensive comparison of all the two-phase pressure drop and flow boiling heat transfer prediction methods available in the open literature is also performed versus the large diversified database presented in Part 1. The experimental databank, from numerous independent research studies, is then utilized to develop the new prediction methods to evaluate local heat transfer coefficients and pressure drops. These new methods were developed from 1903 heat transfer and 1513 frictional pressure drop data points (3416 total), respectively, and were proved to work better over a very wide range of operating conditions, plate designs and fluids (including ammonia). The prediction for flow boiling heat transfer coefficients was broken down into separate macro- and microscale methods.
\end{abstract}

\section{Keywords}

Dimensional analysis

Flow Boiling

General heat transfer prediction method

General pressure drop prediction method

Multiple regression technique

Plate heat exchangers

\section{Contents}

Nomenclature

Introduction

Sensitivity analysis

Comparison of prediction methods

Statistical comparison of prediction methods to data

Dimension analysis and new flow boiling heat transfer and pressure drop prediction methods

Conclusions

Conflict of interests

References 


\begin{tabular}{|c|c|c|c|}
\hline \multicolumn{4}{|c|}{ Nomenclature } \\
\hline $\mathrm{b}$ & mean spacing between two plates (m) & $\lambda$ & percentage predicted within $\pm 50 \%$ \\
\hline Bo & Boiling number & $\Lambda$ & wavelength of surface corrugation (m) \\
\hline $\mathrm{Bd}$ & Bond number & $\mu$ & dynamic viscosity (Pa s) \\
\hline $\mathrm{C}$ & leading coefficient for prediction methods & $\rho$ & density $\left(\mathrm{kgm}^{-3}\right)$ \\
\hline d & diameter $(\mathrm{m})$ & $\rho^{*}$ & density ratio \\
\hline $\mathrm{f}$ & Fanning friction factor & $\sigma$ & surface tension $\left(\mathrm{Nm}^{-1}\right)$ \\
\hline g & acceleration due to the gravity $\left(\mathrm{m}^{2} \mathrm{~s}^{-1}\right)$ & $\varphi$ & enlargement factor \\
\hline G & mass flux $\left(\mathrm{kgm}^{-2} \mathrm{~s}^{-1}\right)$ & $\tau$ & shear stress $\left(\mathrm{Nm}^{-2}\right)$ \\
\hline $\mathrm{h}$ & heat transfer coefficient $\left(\mathrm{Wm}^{-2} \mathrm{~K}^{-1}\right)$ & Y & vector of data \\
\hline $\mathrm{i}_{\mathrm{lv}}$ & latent heat of vaporization $\left(\mathrm{Jkg}^{-1}\right)$ & $\xi$ & percentage predicted within $\pm 30 \%$ \\
\hline $\mathrm{k}$ & thermal conductivity $\left(\mathrm{Wm}^{-1} \mathrm{~K}^{-1}\right)$ & $\Pi$ & dimensionless number \\
\hline $\mathrm{L}$ & length (m) & $\Psi$ & dimensionless number \\
\hline $\mathrm{n}$ & number of data & & Subscripts \\
\hline $\mathrm{Nu}$ & Nusselt number & eq & equivalent \\
\hline $\mathrm{p}$ & pressure $(\mathrm{Pa})$ & $\exp$ & experimental \\
\hline q & heat flux $\left(\mathrm{Wm}^{-2}\right)$ & fri & frictional \\
\hline $\operatorname{Re}$ & Reynolds number & $\mathrm{h}$ & hydraulic \\
\hline $\mathrm{T}$ & temperature $(\mathrm{K})$ & 1 & liquid \\
\hline $\mathrm{u}_{\mathrm{s}}$ & superficial velocity $\left(\mathrm{ms}^{-1}\right)$ & lo & liquid only \\
\hline $\mathrm{W}$ & width (m) & $\mathrm{m}$ & mean or homogeneous \\
\hline $\mathrm{We}$ & Weber number & $\mathrm{p}$ & port \\
\hline \multirow[t]{2}{*}{$\mathrm{X}$} & vapor quality & pre & predicted \\
\hline & Greek symbols & $\mathrm{r}$ & reduced \\
\hline$\beta$ & chevron angle $\left(^{\circ}\right)$ & sat & saturation \\
\hline$\beta^{*}$ & reduced chevron angle & tp & two phase \\
\hline$|\delta|$ & mean absolute error & $\mathrm{v}$ & vapor \\
\hline$\delta$ & mean error & vo & vapor only \\
\hline$\Delta$ & difference & wall & wall \\
\hline
\end{tabular}

\section{Introduction}

In the first part of the present work, an overview of the main research studies was given, the numerous prediction methods proposed in the literature to evaluate heat transfer coefficients and pressure drops were listed, and finally a comprehensive flow boiling databank was collected (see Table 1, Part 1). Based on these results, in the current paper, the goal is to make a detailed comparison of the most quoted prediction methods against one another and perform a statistical comparison between the experimental databases (collected from the literature) and the prediction methods in order to analyze their accuracies. Finally, new prediction methods for the thermal-hydraulic performance of PHE's will be proposed here that provide better accuracies than the previous methods, in addition to covering a wider range of operating conditions, fluids and plate geometries.

\section{Sensitivity analysis}

The performance of the plate heat exchangers is strongly dependent on the geometry and dimensions of the corrugations of the plates. Therefore, it is instructive to perform a sensitivity analysis in order to highlight these influences. Accordingly, the heat transfer and frictional pressure drop prediction methods, described in Part 1, are used here to assess the effect of plate geometry and dimensions on thermal-hydraulic performance. 
The influence of the chevron angle, $\beta$, and wavelength of surface corrugation, $\Lambda$, on the flow boiling heat transfer coefficient and the pressure drop are respectively illustrated in Fig. 1 and Fig. 2. For the following sensitivity analysis, the correlations are evaluated assuming saturated flow boiling of R410A, for the vapor quality range from 0.1 to 0.90 and the following typical conditions: a mass flux equal to $27 \mathrm{~kg} \mathrm{~m}^{-2} \mathrm{~s}^{-1}$, a heat flux of $5.5 \mathrm{~kW} \mathrm{~m}^{-2}$, a saturation temperature of $15^{\circ} \mathrm{C}$, a plate width of $200 \mathrm{~mm}$ and a port to port length of $600 \mathrm{~mm}$. The Han et al. (2003b) correlation was chosen for this parametric study. It can be observed that the heat transfer coefficient, as well as the associated frictional pressure drop, increases with vapor quality along the plate in the convective flow boiling regime. In fact, during the evaporation process the refrigerant vapor quality grows and thus the specific volume grows and consequently the fluid velocity rises. The larger velocity promotes more shear between the liquid and vapor phases and provides higher turbulence; thus, the convective heat transfer coefficient is enhanced and the associated frictional pressure drop grows as well.

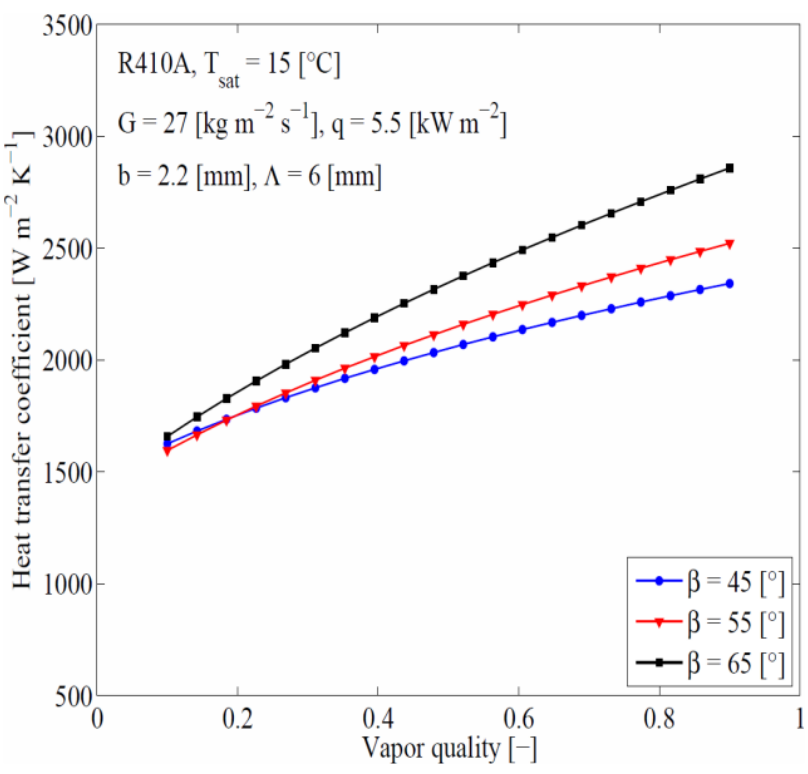

(a)

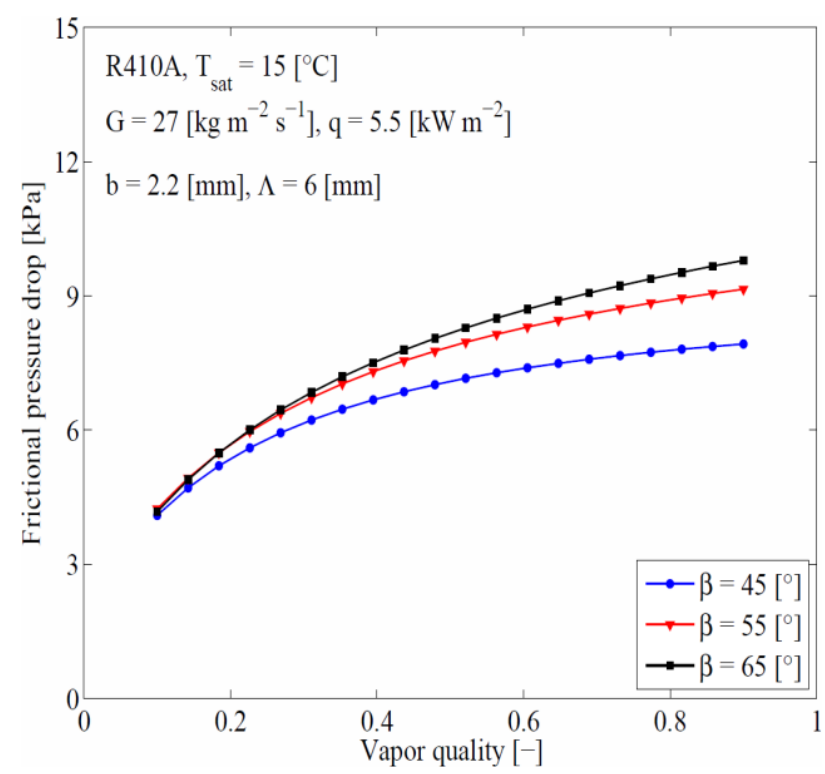

(b)

Fig. 1: Sensitivity analysis on the effect of chevron angle, $\beta$, on: (a) heat transfer coefficient, (b) frictional pressure drop, using the correlations proposed by Han et al. (2003b).

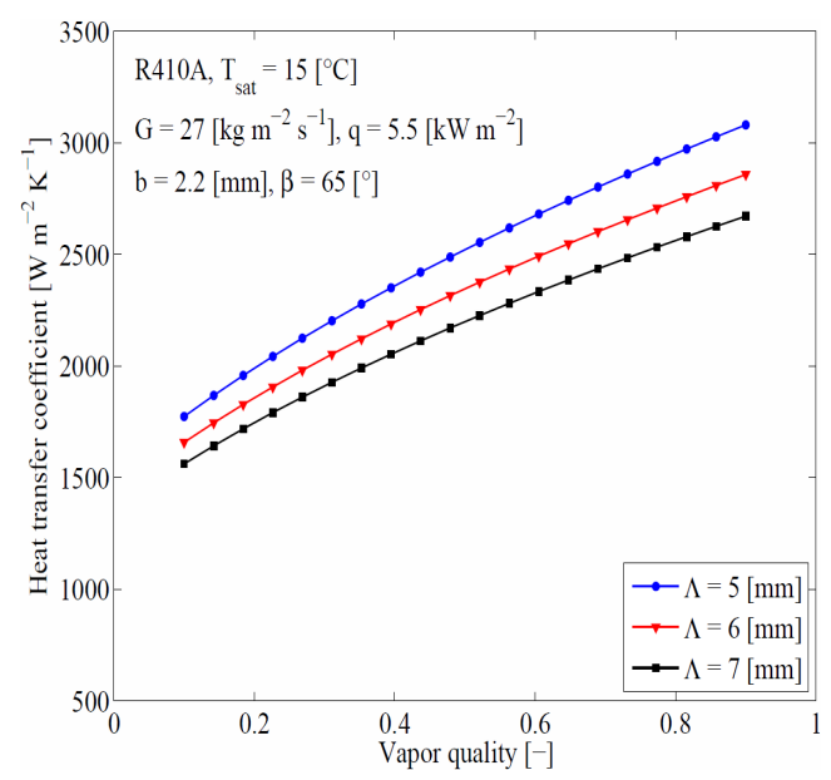

(a)

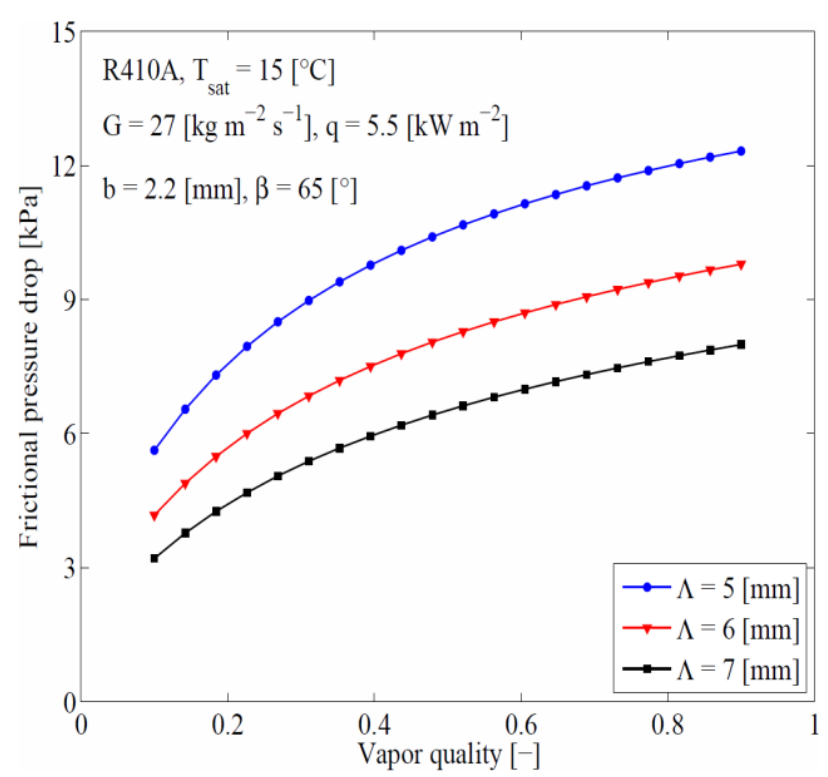

(b)

Fig. 2: Sensitivity analysis on the effect of wavelength of surface corrugation, $\Lambda$, on: (a) heat transfer coefficient, (b) frictional pressure drop, using the correlations provided by Han et al. (2003b). 
It can be seen that at the same value of vapor quality, the higher the plate chevron angle, the more effective the heat transfer but the higher the pressure drop (shown in Fig. 1a and Fig. 1b). Similar outcomes are achieved for the shorter corrugation pitches, i.e. the higher aspect ratios (see Fig. 2a and Fig. 2b). Eventually at high values of $\beta$, as well as at low $\Lambda$, the thermal boundary layer is more frequently interrupted; also, the cross and swirl flows pass around crest of the plate and furrows, producing more turbulence.

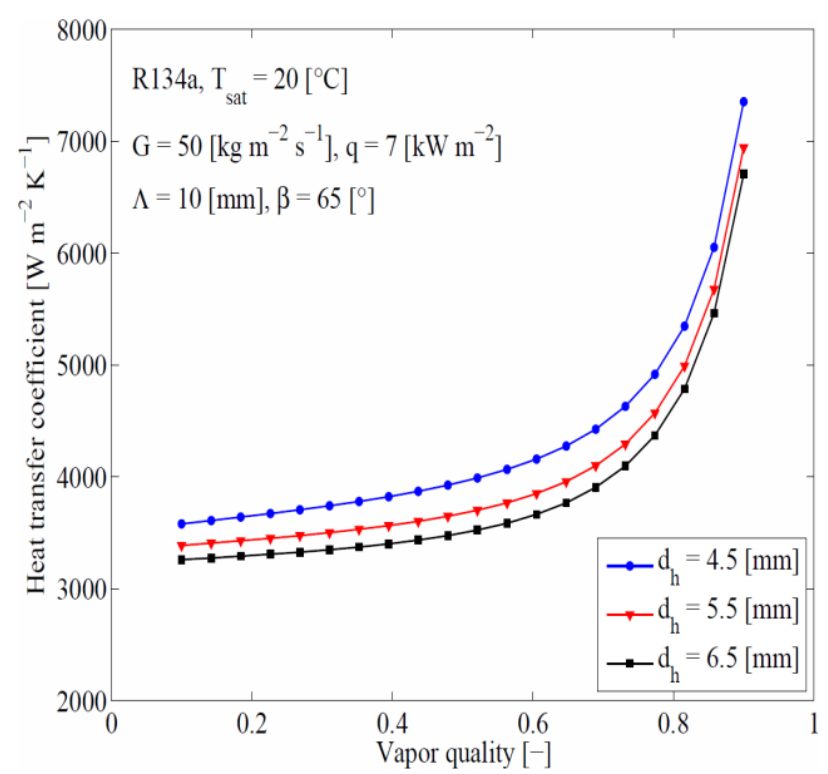

(a)

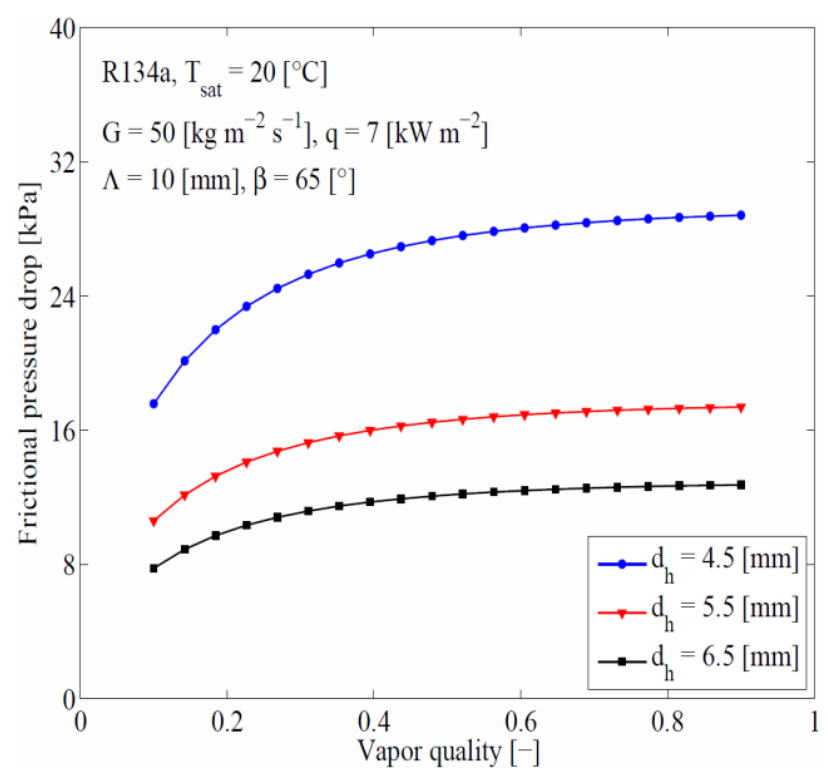

(b)

Fig. 3: Sensitivity analysis on the effect of hydraulic diameter, $d_{h}$, on (a) heat transfer coefficient and (b) frictional pressure drop, using the correlations proposed by Hsieh and Lin (2003c).

Similarly, the effect of hydraulic diameter on the thermal-hydraulic performance is presented in Fig. 3, using the Hsieh and Lin (2003c) correlations for saturated flow boiling of R134a, for the vapor quality range from 0.1 to 0.90 and the following typical conditions: a mass flux equal to $50 \mathrm{~kg} \mathrm{~m}^{-2} \mathrm{~s}^{-1}$, a heat flux of $7 \mathrm{~kW} \mathrm{~m}^{-2}$, a saturation temperature of $20^{\circ} \mathrm{C}$, a plate width of $200 \mathrm{~mm}$ and a port to port length of $600 \mathrm{~mm}$. As can be observed, the heat transfer coefficient increases and the pressure drop rises even much more when decreasing the hydraulic diameter. This is due to the fact that the pressure drop, heat transfer coefficient, and the Nusselt number are inversely proportional to the hydraulic diameter. Normally, in practice, this would suggest a different plate length to width ratio, to reduce the pressure drop, while also taking the PHE design towards more, but smaller plates.

In Part 1, the state-of-the-art for evaporation in plate heat exchangers was presented. In many of these studies, correlations have been proposed to predict two-phase heat transfer coefficients and pressure drops in chevron PHEs. Although the majority of equations are study-specific since they are related to one specific geometry, one test fluid and a limited experimental range, nevertheless it is instructive to discuss and contrast some of their predictions; this will be done in the section below.

\section{Comparison of prediction methods}

In order to highlight the behavior and relative predictions of the different methods, several flow boiling models have been compared for a particular operating condition, which inevitably involves extrapolation of some of these methods beyond their original test conditions (see Fig. 4). To perform such a comparison, the correlations are evaluated for the case of saturated flow boiling at the following nominal test conditions: range of inlet to outlet vapor quality from 0.1 to 0.9 , mass flux of $50 \mathrm{~kg} \mathrm{~m}^{-2} \mathrm{~s}^{-1}$, heat flux of $20 \mathrm{~kW} \mathrm{~m}^{-2}$, saturation temperature of $25^{\circ} \mathrm{C}$, chevron angle of $65^{\circ}$, corrugation pitch of $5 \mathrm{~mm}$, enlargement factor of 1.27 , hydraulic diameter of $5 \mathrm{~mm}$, a plate width of $200 \mathrm{~mm}$, a port to port length of $600 \mathrm{~mm}$ and refrigerant R134a. 


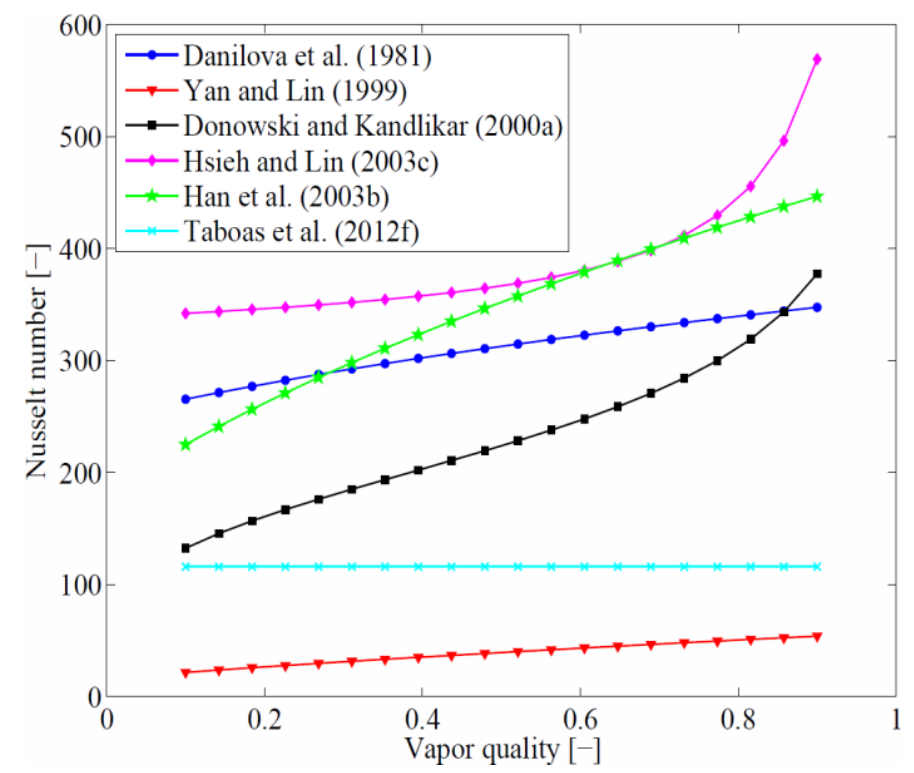

Fig. 4: Predicted Nusselt number against vapor quality for several flow boiling prediction methods.

Concerning Fig. 4, Danilova et al. (1981) observed that the evaporation heat transfer coefficient increased almost linearly with the vapor quality and mass flux due to the dominant contribution of the convective boiling mechanism. Yan and Lin (1999) found that the heat transfer rate was strongly depending on the mass flux and vapor quality. At low vapor quality $(\mathrm{x}<0.45)$, the refrigerant mass flux did not play as important a role because of the lower convection effect on the heat transfer. As soon as the vapor quality became greater than 0.45 , the effect of the mass velocity was seen to be more important. In these conditions, an acceleration of the flow can be observed, which provided higher interfacial shear forces as well as high turbulence in the flow that enhanced the heat transfer coefficient. Obviously, one can see that this correlation underestimated the evaporation heat transfer compared to the other methods over the entire range of vapor qualities. Donowski and Kandlikar (2000a) also surmised that the evaporation process was convective boiling dominated and as a consequence the predicted Nusselt number was still strongly dependent on the vapor quality. Hsieh and Lin (2003c) recognized that depending on the test conditions, nucleate boiling regime was dominant at low vapor fraction region, consequently the Nusselt number was almost independent of the vapor quality and mass flux. On the contrary, the convective flow boiling regime became more important at high values of vapor quality. Han et al. (2003b) concluded that the heat transfer coefficient increased with vapor quality for the entire experimental range, mainly due to strong turbulence in the flow due to the complex flow passage inside plate heat exchangers. The Taboas et al. (2012f) established a criteria in order to decide which mechanism is more effective between nucleate and convective boiling in the evaporation heat transfer rate. According to the above test conditions, the Nusselt number was regardless of vapor quality because the heat transfer rate was nucleate boiling governed. Finally, the highest value of the Nusselt number is predicted by the Hsieh and Lin correlation (2003c). On the contrary, and the lowest heat transfer rate is predicted using correlation of Yan and Lin (1999). However, the order may change as the operating test condition changes. The variation is very wide, where the highest Nusselt number prediction method is more than 10 times that of the lowest. Even excluding the lower two methods, the spread in the predictions is very large. In brief, these methods have huge differences when evaluated for the most commonly used refrigerant, R134a.

Fig. 5 illustrates the trends of frictional pressure gradient versus vapor quality for several empirical correlations, evaluated at the designated operating condition above. Yan and Lin (1999) recognized that the frictional pressure drop grew with a rising of vapor quality. Moreover, when the vapor fraction became greater than 0.72 , the frictional pressure gradient increased linearly with vapor quality and this behavior was justified because of the transition between laminar and turbulent flow regime. Longo and Gasparella (2007c) reported that the frictional pressure gradient was independent of the vapor quality, since the friction factor was found to be dependent only on the wall roughness, similar to what is observed in case of single-phase fully developed turbulent flow. In any case, the authors investigated complete evaporation within their plate heat exchanger, thus the mean vapor quality was equal to 0.5 returning a constant value of 
two-phase density. Hsieh and Lin (2003c, 2002b) and Han et al. (2003b) found that the frictional pressure drop increased with vapor quality but for relatively high vapor fraction was flat or decreased. Moreover, in the these studies no transition was observed from a laminar to a turbulent flow regime. Huang et al. (2012a) found that the frictional pressure drop rose linearly with the vapor quality and this trend may be due to the fact that the prediction method was extrapolated to a relatively high value of mass flux. In summary, the highest frictional pressure gradient was predicted using the model proposed by Longo and Gasparella (2007c), which was developed from mean data so it could not predict the local trends. Instead, the lowest value was estimated adopting the correlation suggested by Han et al. (2003b), giving values only about one-fourth of the former one. There are three methods (the $2^{\text {nd }}, 3^{\text {rd }}$ and $4^{\text {th }}$ from the top) that give reasonably similar values and trends.

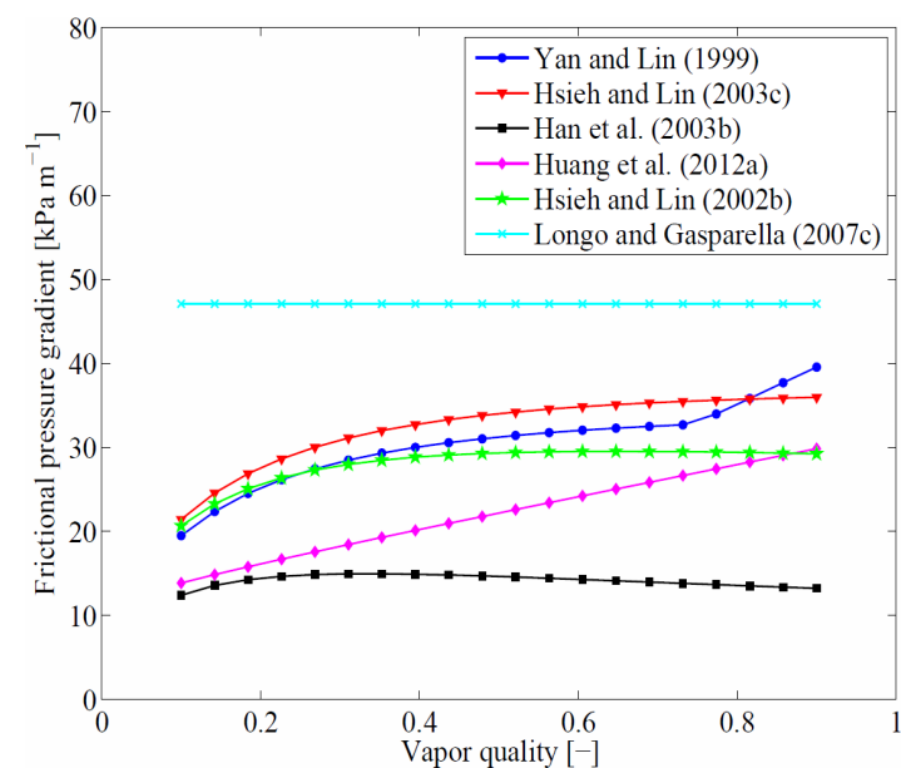

Fig. 5: Predicted frictional pressure gradient against vapor quality for several flow boiling prediction methods.

\section{Statistical comparison of prediction methods to data}

The correlations cited here have each been proposed on rather limited data sets (usually only their own database) and for a specific geometry, testing fluid and limited experimental range; thus, the next step is to compare them to a larger database and a broader range of applicability. Therefore, the comprehensive databank collected in Part 1, consisting of evaporation experimental data of PHE's from numerous independent research studies, will be used to assess the existing PHE's two-phase prediction methods.

This experimental databank for plate heat exchangers was built up including the flow boiling heat transfer and frictional pressure drop data published by thirteen research groups in the literature. In the present study, 3416 data points have been collected and they were gathered for evaporation heat transfer (1903 data) and pressure drop (1513 data) studies in PHEs, including saturated, adiabatic and subcooled flow boiling conditions (i.e. Ouazia (2001a) and group of Lin (2002a)), several kind of heat exchangers (gasketed, brazed, welded and shell and plate), several flow arrangements (parallel/counter flow as well as upward/downward flow), different heat sources (electrical heating, adiabatic and secondary fluid), pure fluids (R134a, ammonia, R245fa, R236fa, R600a, R290, R1270, R1234yf) and several mixtures (among which R410A, R507A, ammonia/water, air/water) for hydraulic diameters ranging from $1.7 \mathrm{~mm}$ to $8.0 \mathrm{~mm}$, chevron angles from $27^{\circ}$ to $70^{\circ}$ and operating conditions of saturation temperatures from -25 to $39^{\circ} \mathrm{C}$, mass fluxes from 5.5 to $610 \mathrm{~kg} \mathrm{~m}^{-2} \mathrm{~s}^{-1}$, heat fluxes from 0.1 to $50.0 \mathrm{~kW} \mathrm{~m}^{-2}$ and vapor qualities from 0 to 0.95 . See Table 1 in Part 1 to find more details of these experimental studies, geometry specifications, and the range of experiments.

In this section, a statistical comparison in terms of heat transfer and pressure drop of the experimental data points for the evaporation processes will be performed against the prediction methods. This statistical analysis will be based on several pertinent statistical parameters: mean error, mean absolute error, percentage of the data points within $\pm 30 \%$ and 
$50 \%$ of the predicted values ${ }^{1}$. In particular, 29 empirical correlations were evaluated (Nusselt numbers and frictional pressure gradients) versus the collected databank. In order to evaluate the prediction methods at any individual operating condition, the following assumptions have been made for which the experimental data points were obtained:

- In the case that the surface enlargement factor was not specified, the hydraulic diameter was evaluated as the equivalent diameter, thus two times the mean spacing between the plates.

- Since in several articles some relevant parameters are missing (e.g., inlet/exit vapor quality, or heat flux, or wall temperature), in these cases the data were reprocessed to determine the missing parameters based on the available information presented in the corresponding article.

- Some prediction methods require the temperature difference between wall and refrigerant. The value has been quantified as the ratio of the average imposed heat flux to the corresponding experimental heat transfer coefficient, i.e. by applying Newton's law of cooling.

- In the definition of the modified Jacob number, Hsieh et al. (2002a) took into account the cross-channel superheat, characterized as the temperature difference between the water and refrigerant streams. First, it has to be noted that this is different compared to the classical definition of the Jacob number in which the wall to refrigerant temperature difference is used. Since the publication did not provide sufficient information about the secondary fluid, in the present analysis the corresponding temperature difference was assumed as two times the wall to refrigerant temperature difference (i.e. the thermal resistances were assumed to be approximately equivalent in both streams).

- Several prediction methods were developed based on the mean value of complete evaporation of the fluid. In this case, the mean refrigerant vapor quality of $x_{m}=0.5$ was used in order to evaluate the correlations. These prediction models are not of course able to predict the local trend of heat transfer coefficient with vapor quality.

- As mentioned in the previous section, the Khan et al. (2012b) correlation to predict Fanning friction factor in the plate of $30^{\circ}$ chevron angle has been disqualified, as it is not able to reproduce the experimental data presented in their article. Hence, to perform the comparison, the Khan et al. (2014b) correlation for the mixed arrangement of $45^{\circ}$ chevron angle was also applied to the test condition for which the chevron angle was below $45^{\circ}$.

- The thermal performance estimated by the Huang et al. (2012a) model were scaled up to be compared in terms of hydraulic diameter, since the authors considered the bubble departure diameter as characteristic length in the expression of the Nusselt number.

- The pressure drop across the inlet and outlet header was quantified applying the scheme suggested by Shah and Focke (1988) (see eq. 11 in Part 1). In this equation, the mass flux was calculated based on the cross section of the ports, while the fluid density was estimated according to the state of the refrigerant. Since, the authors developed the correlation only for single phase experimental data, the use of the model for two-phase flow, simply replacing the liquid with homogeneous two-phase density, represents an extrapolation. As a consequence, the heat transfer coefficient and the associated frictional pressure drop could be underestimated.

In order to categorically assess the prediction methods, they were separately compared to the experimental data of each individual research group. The results of statistical analysis are summarized in Table 1 and Table 2, where each row represents one prediction method compared to the experimental data collected from each individual research group listed in the columns. Accordingly, some of the prediction methods were also validated against their original experimental data (shown in bold) if some experimental data from this group were included in the databank. Furthermore, it has to be pointed out that some prediction methods were not included in the analysis because the authors did not mention in their papers the relevant single phase heat transfer or pressure drop method to use in their prediction methods: Ouazia (2001a), Palm and Claesson (2006b) and Müller and Steinhagen (2001b, 2001c). Finally, the database provided by Müller and

\footnotetext{
${ }^{1}$ The statistical parameters are defined as:

$\delta=\frac{100}{n} \sum_{i=1}^{n} \frac{Y_{i, p r e}-Y_{i, \exp }}{Y_{i, \text { exp }}}, \quad|\delta|=\frac{100}{n} \sum_{i=1}^{n}\left|\frac{Y_{i, p r e}-Y_{i, \exp }}{Y_{i, \exp }}\right|, \quad \xi=n \pm 30 \%, \quad \lambda=n \pm 50 \%$
} 
Steinhagen (2001b, 2001c) and Nilpueng and Wongwises (2010a) were not included in the present study, since the authors experimentally investigated adiabatic two-phase pressure drops of air/water within PHEs.

In Table 1, according to the statistical parameters defined earlier, the comparison between the experimental heat transfer database and the associated prediction methods is shown. The Danilova et al. (1981) method provided the best prediction for the entire databank, most likely due to the fact that their method was developed based on several different plate evaporators and several refrigerants. Their correlation takes into account several important parameters to characterize the heat transfer rate: the vapor Reynolds number quantifying the shear stress between the liquid film and vapor phase, the Bond number accounting for the change in heat transfer phenomena when going from macro-to-micro scale (of course, this was not said at the time) and the Boiling number to include the effect of heat flux on the heat transfer rate. As expected, The Yan and Lin (1999) correlation underpredicted the entire experimental database. Downoski and Kandlikar (2000a) suggested a new prediction correlation based on Yan and Lin (1999) dataset, which was able to predict within a reasonable range the experimental data bank. The Hsieh and Lin (2002b) method was found to slightly overestimate the entire dataset mainly due to the fact that the heat transfer coefficient was only linked to the nucleate boiling regime. Better agreement was achieved using their second prediction method (2003c), which included the contributions of the nucleate and convective terms. In fact, the later correlation takes into account the effects of heat flux and mass flux, vapor quality, and fluid properties; thus, it provided better prediction capability compared to the earlier correlation. The Sterner and Sunden (2006c) and Park and Kim (2004) methods largely overestimated all the datasets, especially the latter method because shell and plate evaporators provide higher heat transfer rates than the common plate heat exchangers (gasketed, brazed welded/semi-welded). Lee et al. (2014a) correlations poorly predicted the experimental databank and the two methods showed good agreement only against their own data. The reason can be explained since the Lee et al. (2014a) method was developed for flow boiling of water at low mass fluxes, which exhibits different behavior compared to refrigerants. The model introduced by Huang et al. (2012a) predicted most of the experimental databank, except the database collected by Khan et al. (2014b, 2012b, 2012c) and Park and Kim (2004). Moreover, it has to be noted that, one group, $i_{1 v} \cdot d_{0} / \alpha^{2}$, (see Table 3, Part 1), presented in their model was not dimensioneless. The Taboas et al. (2012f) method could not offer an appropriate agreement for the other datasets, since it was developed for a low range of the vapor quality $(x<0.2)$ and for water/ammonia mixtures, and thus it was affected by mass transfer due to the temperature glide of water/ammonia mixture. The Khan et al. (2014b, 2012c) correlation, based on ammonia data, was able to predict only their own experimental dataset, and it basically tends to underestimate the data from the other groups when extrapolated to these other refrigerants. Ayub (2003a) suggested an empirical correlation for a large databank including R22 and ammonia, taking into account the effects of port to port length, chevron angle, fluid properties and mass flux on the heat transfer coefficient. Even so, this method predicted the present databank with only a marginal success. Palm and Claesson (2006b) proposed using a Cooper (1984) correlation scheme scaled up by a factor of 1.5 in order to take into account the enhanced heat transfer that can be achieved in the PHEs compared to the plain channels. The Palmer et al. (2000b) correlation (for this analysis, only the first scheme was used) predicted most of the data with a discrete agreement, but the method did not predict the dataset of Taboas et al. (2010b) and Huang et al. (2012a) because of the fluid properties of the ammonia/water and R507A mixtures. The Jokar et al. (2006a) prediction method could not predict any of the experimental database proposed except those of Han et al. (2003b) and Lee et al. (2014a). The Han et al. (2003b) correlation underestimated most of the collected experimental data, since the model was apparently strongly dependent on its original plate geometry. Finally, it has to be highlighted that the present method could not be compared versus the experimental database furnished by Ouazia (2001a) since the author did not mentioned the corrugation pitch of the plate heat exchanger in his paper.

Finally, the best agreements between the predicted and the experimental evaporation heat transfer were achieved by the following six methods: Danilova et al. (1981), Han et al. (2003b), Huang et al. (2012a), Donowski and Kandlikar (2000a) and two by Hsieh and Lin (2003c, 2002b). In Fig. 6 these methods are graphically compared against the current databank (see Table 1, Part 1) and, inside the graph, information on their mean absolute error, number of data points and percentage of the data points within $\pm 50 \%$ are shown. 
Table 1: Two-phase Nusselt numbers predicted by the correlation along the rows compared to the individual experimental database along the columns.

\begin{tabular}{|c|c|c|c|c|c|c|c|c|c|c|c|}
\hline Author(s) & $\begin{array}{l}\text { Lin et al. } \\
\text { (2002a, 2003c, } \\
\text { 2002b, 1999) }\end{array}$ & $\begin{array}{l}\text { Ouazia } \\
\text { (2001a) }\end{array}$ & $\begin{array}{l}\text { Djordjevic and } \\
\text { Kabelac } \\
\text { (2008) }\end{array}$ & $\begin{array}{l}\text { Taboas et al. } \\
\text { (2012f, } \\
\text { 2010b) }\end{array}$ & $\begin{array}{l}\text { Huang et al. } \\
\text { (2012a) }\end{array}$ & $\begin{array}{l}\text { Khan et al. } \\
(2014 b \text {, } \\
\text { 2012b, 2012c) }\end{array}$ & $\begin{array}{l}\text { Lee et al. } \\
\text { (2014a) }\end{array}$ & $\begin{array}{l}\text { Longo et al. } \\
\text { (2012d, } \\
2012 \mathrm{e}, 2007 \mathrm{a} \text {, } \\
2007 \mathrm{~b}, 2007 \mathrm{c})\end{array}$ & $\begin{array}{l}\text { Han et al. } \\
\text { (2003b) }\end{array}$ & $\begin{array}{l}\text { Park and } \\
\text { Kim } \\
(2004)\end{array}$ & $\begin{array}{l}\text { Vakili-Farahani } \\
\text { et al. } \\
\text { (2014c, 2014d) }\end{array}$ \\
\hline $\begin{array}{l}\text { Danilova et al. } \\
\text { (1981) }\end{array}$ & $\begin{array}{l}|\delta|=20.1 \\
\delta=-17.4 \\
\xi=66.1 \\
\lambda=94.6\end{array}$ & $\begin{array}{l}|\delta|=29.1 \\
\delta=29 \\
\xi=51.2 \\
\lambda=75.6\end{array}$ & $\begin{array}{l}|\delta|=18.9 \\
\delta=-15.6 \\
\xi=95.7 \\
\lambda=99.1\end{array}$ & $\begin{array}{l}|\delta|=23.7 \\
\delta=-19.5 \\
\xi=63.9 \\
\lambda=98.7\end{array}$ & $\begin{array}{l}|\delta|=14.7 \\
\delta=14.1 \\
\xi=82.1 \\
\lambda=99.1\end{array}$ & $\begin{array}{l}|\delta|=33.3 \\
\delta=30.3 \\
\xi=73.8 \\
\lambda=87.9\end{array}$ & $\begin{array}{l}|\delta|=56.1 \\
\delta=-34 \\
\xi=11.3 \\
\lambda=24.2\end{array}$ & $\begin{array}{l}|\delta|=29.4 \\
\delta=20.7 \\
\xi=59 \\
\lambda=82.1\end{array}$ & $\begin{array}{l}|\delta|=49.2 \\
\delta=38 \\
\xi=48.1 \\
\lambda=67.3\end{array}$ & $\begin{array}{l}|\delta|=27.5 \\
\delta=-27.5 \\
\xi=68.9 \\
\lambda=99.1\end{array}$ & $\begin{array}{l}|\delta|=33.8 \\
\delta=12.6 \\
\xi=67.1 \\
\lambda=86.1\end{array}$ \\
\hline $\begin{array}{l}\text { Yan and Lin } \\
\text { (1999) }\end{array}$ & $\begin{array}{l}|\boldsymbol{\delta}|=\mathbf{9 0 . 4} \\
\boldsymbol{\delta}=\mathbf{- 9 0 . 4} \\
\xi=\mathbf{0} \\
\lambda=\mathbf{0}\end{array}$ & $\begin{array}{l}|\delta|=88.1 \\
\delta=-88.1 \\
\xi=0 \\
\lambda=0\end{array}$ & $\begin{array}{l}|\delta|=78.3 \\
\delta=-78.3 \\
\xi=0 \\
\lambda=0\end{array}$ & $\begin{array}{l}|\delta|=84.4 \\
\delta=-84.4 \\
\xi=0 \\
\lambda=0\end{array}$ & $\begin{array}{l}|\delta|=80.4 \\
\delta=-80.4 \\
\xi=0 \\
\lambda=0\end{array}$ & $\begin{array}{l}|\delta|=45.8 \\
\delta=-44.1 \\
\xi=19.6 \\
\lambda=56.1\end{array}$ & $\begin{array}{l}\delta \mid=68.6 \\
\delta=-61.1 \\
\xi=12.9 \\
\lambda=19.4\end{array}$ & $\begin{array}{l}|\delta|=75.2 \\
\delta=-75.2 \\
\xi=0 \\
\lambda=0\end{array}$ & $\begin{array}{l}|\delta|=79 \\
\delta=-79 \\
\xi=0 \\
\lambda=0\end{array}$ & $\begin{array}{l}|\delta|=89.8 \\
\delta=-89.8 \\
\xi=0 \\
\lambda=0\end{array}$ & $\begin{array}{l}|\delta|=75.1 \\
\delta=-75.1 \\
\xi=0.8 \\
\lambda=2.4\end{array}$ \\
\hline $\begin{array}{l}\text { Hsieh and Lin } \\
(2002 b)\end{array}$ & $\begin{array}{l}|\boldsymbol{\delta}|=\mathbf{3 1 . 5} \\
\delta=\mathbf{2 6 . 6} \\
\xi=\mathbf{5 0} \\
\lambda=\mathbf{8 2 . 5}\end{array}$ & $\begin{array}{l}|\delta|=65.7 \\
\delta=65.7 \\
\xi=48.8 \\
\lambda=51.2\end{array}$ & $\begin{array}{l}|\delta|=26.6 \\
\delta=23.6 \\
\xi=71.3 \\
\lambda=87\end{array}$ & $\begin{array}{l}|\delta|=105.5 \\
\delta=105.5 \\
\xi=1.3 \\
\lambda=7.9\end{array}$ & $\begin{array}{l}|\delta|=23.3 \\
\delta=20.9 \\
\xi=70.1 \\
\lambda=82.9\end{array}$ & $\begin{array}{l}|\delta|=85.5 \\
\delta=85.5 \\
\xi=6.5 \\
\lambda=30.8\end{array}$ & $\begin{array}{l}|\delta|=50 \\
\delta=-32.5 \\
\xi=12.9 \\
\lambda=43.5\end{array}$ & $\begin{array}{l}|\delta|=35.1 \\
\delta=32.2 \\
\xi=55.2 \\
\lambda=74.4\end{array}$ & $\begin{array}{l}|\delta|=49.7 \\
\delta=38.2 \\
\xi=59.6 \\
\lambda=67.3\end{array}$ & $\begin{array}{l}|\delta|=37.5 \\
\delta=-37.5 \\
\xi=28.3 \\
\lambda=86.8\end{array}$ & $\begin{array}{l}|\delta|=39.9 \\
\delta=23.1 \\
\xi=50.8 \\
\lambda=70.3\end{array}$ \\
\hline $\begin{array}{l}\text { Hsieh and Lin } \\
\text { (2003c) }\end{array}$ & $\begin{array}{l}|\delta|=19.5 \\
\delta=17.5 \\
\xi=83.7 \\
\lambda=94.8\end{array}$ & $\begin{array}{l}|\delta|=40 \\
\delta=39.2 \\
\xi=51.2 \\
\lambda=53.7\end{array}$ & $\begin{array}{l}|\delta|=51.1 \\
\delta=51.1 \\
\xi=34.8 \\
\lambda=68.7\end{array}$ & $\begin{array}{l}|\delta|=88.9 \\
\delta=88.9 \\
\xi=8.6 \\
\lambda=23\end{array}$ & $\begin{array}{l}|\delta|=61.6 \\
\delta=61.6 \\
\xi=6 \\
\lambda=46.2\end{array}$ & $\begin{array}{l}|\delta|=209.5 \\
\delta=209.5 \\
\xi=0 \\
\lambda=0\end{array}$ & $\begin{array}{l}|\delta|=67.8 \\
\delta=34.9 \\
\xi=50 \\
\lambda=77.4\end{array}$ & $\begin{array}{l}|\delta|=91.3 \\
\delta=91.3 \\
\xi=12.3 \\
\lambda=21.8\end{array}$ & $\begin{array}{l}|\delta|=89.7 \\
\delta=89.6 \\
\xi=28.8 \\
\lambda=40.4\end{array}$ & $\begin{array}{l}|\delta|=22.4 \\
\delta=-18.8 \\
\xi=80.2 \\
\lambda=100\end{array}$ & $\begin{array}{l}|\delta|=25.6 \\
\delta=12.8 \\
\xi=69.9 \\
\lambda=89.9\end{array}$ \\
\hline $\begin{array}{l}\text { Sterner and } \\
\text { Sunden } \\
(2006 c)\end{array}$ & $\begin{array}{l}|\delta|=3448.4 \\
\delta=3448.4 \\
\xi=0 \\
\lambda=0\end{array}$ & $\begin{array}{l}|\delta|=795.8 \\
\delta=795.8 \\
\xi=0 \\
\lambda=0\end{array}$ & $\begin{array}{l}|\delta|=144.3 \\
\delta=136.6 \\
\xi=19.1 \\
\lambda=33\end{array}$ & $\begin{array}{l}|\delta|=3733.8 \\
\delta=3733.8 \\
\xi=0 \\
\lambda=0\end{array}$ & $\begin{array}{l}|\delta|=433.1 \\
\delta=433.1 \\
\xi=2.6 \\
\lambda=8.5\end{array}$ & $\begin{array}{l}|\delta|=77.3 \\
\delta=-77.3 \\
\xi=0 \\
\lambda=4.7\end{array}$ & $\begin{array}{l}|\delta|=98 \\
\delta=-98 \\
\xi=0 \\
\lambda=0\end{array}$ & $\begin{array}{l}|\delta|=594.2 \\
\delta=580.6 \\
\xi=6.4 \\
\lambda=10.3\end{array}$ & $\begin{array}{l}|\delta|=2086 \\
\delta=2086 \\
\xi=0 \\
\lambda=0\end{array}$ & $\begin{array}{l}|\delta|=267.6 \\
\delta=267.6 \\
\xi=0 \\
\lambda=0\end{array}$ & $\begin{array}{l}|\delta|=77.6 \\
\delta=24.1 \\
\xi=23.6 \\
\lambda=40.9\end{array}$ \\
\hline $\begin{array}{l}\text { Downoski and } \\
\text { Kandlikar } \\
(2000 \mathrm{a})\end{array}$ & $\begin{array}{l}|\delta|=22.8 \\
\delta=-4.8 \\
\xi=69.2 \\
\lambda=94.6\end{array}$ & $\begin{array}{l}|\delta|=50.1 \\
\delta=50.1 \\
\xi=26.8 \\
\lambda=48.8\end{array}$ & $\begin{array}{l}|\delta|=27.5 \\
\delta=22.3 \\
\xi=64.3 \\
\lambda=85.2\end{array}$ & $\begin{array}{l}|\delta|=122.1 \\
\delta=122.1 \\
\xi=0 \\
\lambda=0\end{array}$ & $\begin{array}{l}|\delta|=14.3 \\
\delta=-7.1 \\
\xi=96.6 \\
\lambda=100\end{array}$ & $\begin{array}{l}|\delta|=33 \\
\delta=30.5 \\
\xi=72.9 \\
\lambda=86\end{array}$ & $\begin{array}{l}|\delta|=47.8 \\
\delta=33.9 \\
\xi=74.2 \\
\lambda=77.4\end{array}$ & $\begin{array}{l}|\delta|=25.2 \\
\delta=-2 \\
\xi=64.7 \\
\lambda=90.4\end{array}$ & $\begin{array}{l}|\delta|=60 \\
\delta=48.3 \\
\xi=42.3 \\
\lambda=55.8\end{array}$ & $\begin{array}{l}|\delta|=15.8 \\
\delta=-9.5 \\
\xi=84 \\
\lambda=99.1\end{array}$ & $\begin{array}{l}|\delta|=69.2 \\
\delta=51.1 \\
\xi=36.3 \\
\lambda=54.7\end{array}$ \\
\hline $\begin{array}{l}\text { Palmer et al. } \\
(2000 b)\end{array}$ & $\begin{array}{l}|\delta|=25 \\
\delta=-19 \\
\xi=61.8 \\
\lambda=95.3\end{array}$ & $\begin{array}{l}|\delta|=32.8 \\
\delta=23.6 \\
\xi=58.5 \\
\lambda=75.6\end{array}$ & $\begin{array}{l}|\delta|=29 \\
\delta=18.9 \\
\xi=71.3 \\
\lambda=84.3\end{array}$ & $\begin{array}{l}|\delta|=100.1 \\
\delta=100.1 \\
\xi=0 \\
\lambda=2.6\end{array}$ & $\begin{array}{l}|\delta|=19.1 \\
\delta=13.9 \\
\xi=77.8 \\
\lambda=91.5\end{array}$ & $\begin{array}{l}|\delta|=20.7 \\
\delta=13 \\
\xi=87.9 \\
\lambda=87.9\end{array}$ & $\begin{array}{l}|\delta|=39.7 \\
\delta=-15 \\
\xi=14.5 \\
\lambda=88.7\end{array}$ & $\begin{array}{l}|\delta|=25.4 \\
\delta=-17.3 \\
\xi=63.4 \\
\lambda=91.7\end{array}$ & $\begin{array}{l}|\delta|=37 \\
\delta=12.7 \\
\xi=36.6 \\
\lambda=73.1\end{array}$ & $\begin{array}{l}|\delta|=35.6 \\
\delta=-35.6 \\
\xi=30.2 \\
\lambda=94.3\end{array}$ & $\begin{array}{l}|\delta|=270.2 \\
\delta=269.2 \\
\xi=11.5 \\
\lambda=20\end{array}$ \\
\hline $\begin{array}{l}\text { Han et al. } \\
(2003 b)\end{array}$ & $\begin{array}{l}|\delta|=36 \\
\delta=-36 \\
\xi=25.6 \\
\lambda=95.8\end{array}$ & $\begin{array}{l}|\delta|=- \\
\delta=- \\
\xi=- \\
\lambda=-\end{array}$ & $\begin{array}{l}|\delta|=38.9 \\
\delta=-38.1 \\
\xi=14.8 \\
\lambda=95.7\end{array}$ & $\begin{array}{l}|\delta|=14 \\
\delta=-3.5 \\
\xi=94.1 \\
\lambda=99.3\end{array}$ & $\begin{array}{l}|\delta|=19.5 \\
\delta=-14.2 \\
\xi=71.8 \\
\lambda=100\end{array}$ & $\begin{array}{l}|\delta|=57.2 \\
\delta=55.5 \\
\xi=43 \\
\lambda=68.2\end{array}$ & $\begin{array}{l}|\delta|=57.3 \\
\delta=-18.2 \\
\xi=11.3 \\
\lambda=43.5\end{array}$ & $\begin{array}{l}|\delta|=25.9 \\
\delta=-12 \\
\xi=60.3 \\
\lambda=92.3\end{array}$ & $\begin{array}{l}|\delta|=20.9 \\
\delta=-4.6 \\
\xi=75 \\
\lambda=100\end{array}$ & $\begin{array}{l}|\delta|=42.8 \\
\delta=-42.8 \\
\xi=0 \\
\lambda=95.3\end{array}$ & $\begin{array}{l}|\delta|=31.5 \\
\delta=-9.1 \\
\xi=57.9 \\
\lambda=83.7\end{array}$ \\
\hline $\begin{array}{l}\text { Ayub } \\
(2003 a)\end{array}$ & $\begin{array}{l}|\delta|=30.6 \\
\delta=-19.2 \\
\xi=51.7 \\
\lambda=84.5\end{array}$ & $\begin{array}{l}|\delta|=34.5 \\
\delta=-32.2 \\
\xi=34.1 \\
\lambda=82.9\end{array}$ & $\begin{array}{l}|\delta|=47.3 \\
\delta=-21.2 \\
\xi=34.8 \\
\lambda=52.2\end{array}$ & $\begin{array}{l}|\delta|=431.2 \\
\delta=431.2 \\
\xi=0 \\
\lambda=0\end{array}$ & $\begin{array}{l}|\delta|=61.9 \\
\delta=-61.9 \\
\xi=0.9 \\
\lambda=13.7\end{array}$ & $\begin{array}{l}|\delta|=23.5 \\
\delta=-3.2 \\
\xi=86.9 \\
\lambda=87.9\end{array}$ & $\begin{array}{l}|\delta|=27.8 \\
\delta=8.5 \\
\xi=75.8 \\
\lambda=85.5\end{array}$ & $\begin{array}{l}|\delta|=45.9 \\
\delta=-45.7 \\
\xi=17.3 \\
\lambda=57.7\end{array}$ & $\begin{array}{l}|\delta|=31.7 \\
\delta=-26.1 \\
\xi=34.6 \\
\lambda=100\end{array}$ & $\begin{array}{l}|\delta|=64.6 \\
\delta=-64.6 \\
\xi=0 \\
\lambda=1.9\end{array}$ & $\begin{array}{l}|\delta|=68.8 \\
\delta=17.9 \\
\xi=26 \\
\lambda=47\end{array}$ \\
\hline
\end{tabular}


Table 1: Two-phase Nusselt numbers predicted by the correlation along the rows compared to the individual experimental database along the columns (continued).

\begin{tabular}{|c|c|c|c|c|c|c|c|c|c|c|c|}
\hline Author(s) & $\begin{array}{l}\text { Lin et al. } \\
\text { (2002a, 2003c, } \\
\text { 2002b, 1999) }\end{array}$ & $\begin{array}{l}\text { Ouazia } \\
\text { (2001a) }\end{array}$ & $\begin{array}{l}\text { Djordjevic and } \\
\text { Kabelac (2008) }\end{array}$ & $\begin{array}{l}\text { Taboas et al. } \\
\text { (2012f, } \\
2010 \mathrm{~b})\end{array}$ & $\begin{array}{l}\text { Huang et al. } \\
\text { (2012a) }\end{array}$ & $\begin{array}{l}\text { Khan et al. } \\
\text { (2014b, } \\
2012 b, 2012 c)\end{array}$ & $\begin{array}{l}\text { Lee et al. } \\
\text { (2014a) }\end{array}$ & $\begin{array}{l}\text { Longo et al. } \\
\text { (2012d, } \\
2012 \mathrm{e}, 2007 \mathrm{a}, \\
2007 \mathrm{~b}, 2007 \mathrm{c})\end{array}$ & $\begin{array}{l}\text { Han et al. } \\
\text { (2003b) }\end{array}$ & $\begin{array}{l}\text { Park and } \\
\text { Kim } \\
(2004)\end{array}$ & $\begin{array}{l}\text { Vakili-Farahani } \\
\text { et al. } \\
(2014 c, 2014 d)\end{array}$ \\
\hline $\begin{array}{l}\text { Palm and } \\
\text { Claesson } \\
\text { (2006b) }\end{array}$ & $\begin{array}{l}|\delta|=30.8 \\
\delta=20.1 \\
\xi=56.4 \\
\lambda=95.3\end{array}$ & $\begin{array}{l}|\delta|=37.3 \\
\delta=25.2 \\
\xi=48.8 \\
\lambda=56.1\end{array}$ & $\begin{array}{l}|\delta|=25.9 \\
\delta=-5.3 \\
\xi=79.1 \\
\lambda=94.8\end{array}$ & $\begin{array}{l}|\delta|=64.3 \\
\delta=63.7 \\
\xi=23.7 \\
\lambda=35.5\end{array}$ & $\begin{array}{l}|\delta|=23.2 \\
\delta=10 \\
\xi=73.5 \\
\lambda=81.2\end{array}$ & $\begin{array}{l}|\delta|=103.5 \\
\delta=103.5 \\
\xi=5.6 \\
\lambda=25.2\end{array}$ & $\begin{array}{l}|\delta|=57.6 \\
\delta=-45.4 \\
\xi=12.9 \\
\lambda=22.6\end{array}$ & $\begin{array}{l}|\delta|=50.4 \\
\delta=48.6 \\
\xi=26.3 \\
\lambda=55.1\end{array}$ & $\begin{array}{l}|\delta|=48.9 \\
\delta=36.6 \\
\xi=59.6 \\
\lambda=69.2\end{array}$ & $\begin{array}{l}|\delta|=55.1 \\
\delta=-55.1 \\
\xi=0 \\
\lambda=33\end{array}$ & $\begin{array}{l}|\delta|=91.9 \\
\delta=91.4 \\
\xi=11 \\
\lambda=23.6\end{array}$ \\
\hline $\begin{array}{l}\text { Jokar et al. } \\
\text { (2006a) }\end{array}$ & $\begin{array}{l}|\delta|=55.9 \\
\delta=-55.9 \\
\xi=0 \\
\lambda=27.6\end{array}$ & $\begin{array}{l}|\delta|=54 \\
\delta=-54 \\
\xi=7.3 \\
\lambda=36.6\end{array}$ & $\begin{array}{l}|\delta|=92.8 \\
\delta=35.7 \\
\xi=2.6 \\
\lambda=26.1\end{array}$ & $\begin{array}{l}|\delta|=115.4 \\
\delta=115.4 \\
\xi=2.7 \\
\lambda=6.6\end{array}$ & $\begin{array}{l}|\delta|=22.2 \\
\delta=-20 \\
\xi=65 \\
\lambda=100\end{array}$ & $\begin{array}{l}|\delta|=186.2 \\
\delta=186.2 \\
\xi=0 \\
\lambda=0\end{array}$ & $\begin{array}{l}|\delta|=56.9 \\
\delta=38 \\
\xi=72.6 \\
\lambda=77.4\end{array}$ & $\begin{array}{l}|\delta|=30.5 \\
\delta=-29.6 \\
\xi=43 \\
\lambda=86.5\end{array}$ & $\begin{array}{l}|\delta|=39.2 \\
\delta=-1.7 \\
\xi=36.5 \\
\lambda=80.8\end{array}$ & $\begin{array}{l}|\delta|=72.7 \\
\delta=-72.7 \\
\xi=0 \\
\lambda=0\end{array}$ & $\begin{array}{l}|\delta|=67.4 \\
\delta=41.2 \\
\xi=45.2 \\
\lambda=72.3\end{array}$ \\
\hline $\begin{array}{l}\text { Khan et al. } \\
(2014 b \text {, } \\
2012 b, 2012 c)\end{array}$ & $\begin{array}{l}|\delta|=79.8 \\
\delta=-79.8 \\
\xi=0 \\
\lambda=0\end{array}$ & $\begin{array}{l}|\delta|=81.5 \\
\delta=-81.5 \\
\xi=0 \\
\lambda=0\end{array}$ & $\begin{array}{l}|\delta|=49.4 \\
\delta=-22 \\
\xi=31.3 \\
\lambda=47.8\end{array}$ & $\begin{array}{l}|\delta|=36.6 \\
\delta=-35.6 \\
\xi=34.2 \\
\lambda=78.3\end{array}$ & $\begin{array}{l}|\delta|=23.7 \\
\delta=-15.3 \\
\xi=65 \\
\lambda=99.1\end{array}$ & $\begin{array}{l}|\delta|=16.9 \\
\delta=16.3 \\
\xi=87.9 \\
\lambda=87.9\end{array}$ & $\begin{array}{l}|\delta|=55 \\
\delta=-46.9 \\
\xi=14.5 \\
\lambda=25.8\end{array}$ & $\begin{array}{l}|\delta|=48.8 \\
\delta=-48.8 \\
\xi=16.7 \\
\lambda=53.2\end{array}$ & $\begin{array}{l}|\delta|=43.9 \\
\delta=-24.8 \\
\xi=21.2 \\
\lambda=61.5\end{array}$ & $\begin{array}{l}|\delta|=80.6 \\
\delta=-80.6 \\
\xi=0 \\
\lambda=0\end{array}$ & $\begin{array}{l}|\delta|=265.6 \\
\delta=265.6 \\
\xi=3.9 \\
\lambda=13.2\end{array}$ \\
\hline $\begin{array}{l}\text { Taboas et al. } \\
(2012 \mathrm{f})\end{array}$ & $\begin{array}{l}|\delta|=44.7 \\
\delta=-44.7 \\
\xi=15.5 \\
\lambda=62.6\end{array}$ & $\begin{array}{l}|\delta|=26 \\
\delta=-21.3 \\
\xi=56.1 \\
\lambda=95.1\end{array}$ & $\begin{array}{l}|\delta|=38.9 \\
\delta=-32.7 \\
\xi=25.2 \\
\lambda=87\end{array}$ & $\begin{array}{l}|\boldsymbol{\delta}|=32.2 \\
\delta=32.1 \\
\xi=62.5 \\
\lambda=\mathbf{8 3 . 6}\end{array}$ & $\begin{array}{l}|\delta|=39.5 \\
\delta=-39.5 \\
\xi=20.5 \\
\lambda=81.2\end{array}$ & $\begin{array}{l}|\delta|=36.7 \\
\delta=-25.7 \\
\xi=13.1 \\
\lambda=97.2\end{array}$ & $\begin{array}{l}|\delta|=60.7 \\
\delta=-47.2 \\
\xi=11.3 \\
\lambda=25.8\end{array}$ & $\begin{array}{l}|\delta|=49.2 \\
\delta=-49.2 \\
\xi=10.9 \\
\lambda=46.2\end{array}$ & $\begin{array}{l}|\delta|=37.7 \\
\delta=-31.6 \\
\xi=30.8 \\
\lambda=65.4\end{array}$ & $\begin{array}{l}|\delta|=64.6 \\
\delta=-64.6 \\
\xi=0 \\
\lambda=0\end{array}$ & $\begin{array}{l}|\delta|=57.9 \\
\delta=23.5 \\
\xi=34.5 \\
\lambda=58.4\end{array}$ \\
\hline $\begin{array}{l}\text { Huang et al. } \\
\text { (2012a) }\end{array}$ & $\begin{array}{l}|\delta|=42.3 \\
\delta=-41.1 \\
\xi=12.8 \\
\lambda=73.4\end{array}$ & $\begin{array}{l}|\delta|=30.9 \\
\delta=8.1 \\
\xi=48.8 \\
\lambda=95.1\end{array}$ & $\begin{array}{l}|\delta|=28.9 \\
\delta=-19 \\
\xi=48.7 \\
\lambda=96.5\end{array}$ & $\begin{array}{l}|\delta|=30.8 \\
\delta=-29.1 \\
\xi=51.3 \\
\lambda=84.2\end{array}$ & $\begin{array}{l}|\boldsymbol{\delta}|=\mathbf{6 . 8} \\
\delta=\mathbf{- 1 . 7} \\
\xi=\mathbf{1 0 0} \\
\lambda=100\end{array}$ & $\begin{array}{l}|\delta|=70.4 \\
\delta=70.3 \\
\xi=20.6 \\
\lambda=43\end{array}$ & $\begin{array}{l}|\delta|=53.4 \\
\delta=-13.9 \\
\xi=14.5 \\
\lambda=62.9\end{array}$ & $\begin{array}{l}|\delta|=32.3 \\
\delta=3.4 \\
\xi=46.8 \\
\lambda=84\end{array}$ & $\begin{array}{l}|\delta|=36.4 \\
\delta=-27.8 \\
\xi=28.8 \\
\lambda=65.4\end{array}$ & $\begin{array}{l}|\delta|=54.4 \\
\delta=-54.4 \\
\xi=0 \\
\lambda=35.8\end{array}$ & $\begin{array}{l}|\delta|=26.3 \\
\delta=11.5 \\
\xi=70.8 \\
\lambda=90.2\end{array}$ \\
\hline $\begin{array}{l}\text { Park and Kim } \\
\text { (2004) }\end{array}$ & $\begin{array}{l}|\delta|=15.1 \\
\delta=1.7 \\
\xi=91.1 \\
\lambda=99\end{array}$ & $\begin{array}{l}|\delta|=36.5 \\
\delta=34.2 \\
\xi=48.8 \\
\lambda=68.3\end{array}$ & $\begin{array}{l}|\delta|=162.8 \\
\delta=162.8 \\
\xi=0 \\
\lambda=1.7\end{array}$ & $\begin{array}{l}|\delta|=153.7 \\
\delta=153.7 \\
\xi=0 \\
\lambda=0\end{array}$ & $\begin{array}{l}|\delta|=145.5 \\
\delta=145.5 \\
\xi=0 \\
\lambda=0\end{array}$ & $\begin{array}{l}|\delta|=327.7 \\
\delta=327.7 \\
\xi=0 \\
\lambda=0\end{array}$ & $\begin{array}{l}|\delta|=241.9 \\
\delta=241.9 \\
\xi=0 \\
\lambda=0\end{array}$ & $\begin{array}{l}|\delta|=105.8 \\
\delta=105.1 \\
\xi=19.2 \\
\lambda=26.3\end{array}$ & $\begin{array}{l}|\delta|=130 \\
\delta=130 \\
\xi=9.6 \\
\lambda=25\end{array}$ & $\begin{array}{l}|\delta|=11.4 \\
\delta=10.9 \\
\xi=100 \\
\lambda=100\end{array}$ & $\begin{array}{l}|\delta|=626.6 \\
\delta=626.6 \\
\xi=0 \\
\lambda=0\end{array}$ \\
\hline $\begin{array}{l}\text { Lee et al. } \\
\text { (2014a) }\end{array}$ & $\begin{array}{l}|\delta|=50.2 \\
\delta=-48.1 \\
\xi=23.9 \\
\lambda=47.5\end{array}$ & $\begin{array}{l}|\delta|=50.7 \\
\delta=-50.5 \\
\xi=14.6 \\
\lambda=43.9\end{array}$ & $\begin{array}{l}|\delta|=118.5 \\
\delta=98.8 \\
\xi=33 \\
\lambda=49.6\end{array}$ & $\begin{array}{l}|\delta|=100.8 \\
\delta=100.7 \\
\xi=9.2 \\
\lambda=20.4\end{array}$ & $\begin{array}{l}|\delta|=97.3 \\
\delta=97.1 \\
\xi=12 \\
\lambda=24.8\end{array}$ & $\begin{array}{l}|\delta|=121.9 \\
\delta=121.3 \\
\xi=22.4 \\
\lambda=33.6\end{array}$ & $\begin{array}{l}|\delta|=10.6 \\
\delta=9.3 \\
\xi=95.2 \\
\lambda=100\end{array}$ & $\begin{array}{l}|\delta|=48.9 \\
\delta=-45.7 \\
\xi=18.6 \\
\lambda=46.8\end{array}$ & $\begin{array}{l}|\delta|=62.1 \\
\delta=1.3 \\
\xi=36.5 \\
\lambda=48.1\end{array}$ & $\begin{array}{l}|\delta|=62.8 \\
\delta=-62.4 \\
\xi=9.4 \\
\lambda=35.8\end{array}$ & $\begin{array}{l}|\delta|=975 \\
\delta=975 \\
\xi=0 \\
\lambda=0\end{array}$ \\
\hline $\begin{array}{l}\text { Present } \\
\text { method }\end{array}$ & $\begin{array}{l}|\delta|=11.8 \\
\delta=-7.4 \\
\xi=95.6 \\
\lambda=99.3\end{array}$ & $\begin{array}{l}|\delta|=21.3 \\
\delta=0.8 \\
\xi=80.5 \\
\lambda=100\end{array}$ & $\begin{array}{l}|\delta|=22.1 \\
\delta=-21 \\
\xi=91.3 \\
\lambda=100\end{array}$ & $\begin{array}{l}|\delta|=20.3 \\
\delta=6.5 \\
\xi=75.7 \\
\lambda=95.4\end{array}$ & $\begin{array}{l}|\delta|=17.4 \\
\delta=-2.2 \\
\xi=89.7 \\
\lambda=99.2\end{array}$ & $\begin{array}{l}|\delta|=16 \\
\delta=-3.1 \\
\xi=85 \\
\lambda=100\end{array}$ & $\begin{array}{l}|\delta|=60.2 \\
\delta=-50.3 \\
\xi=14.5 \\
\lambda=25.8\end{array}$ & $\begin{array}{l}|\delta|=17 \\
\delta=9.7 \\
\xi=81.4 \\
\lambda=96.7\end{array}$ & $\begin{array}{l}|\delta|=46.7 \\
\delta=40.5 \\
\xi=55.8 \\
\lambda=67.3\end{array}$ & $\begin{array}{l}|\delta|=48.6 \\
\delta=-48.6 \\
\xi=0 \\
\lambda=68\end{array}$ & $\begin{array}{l}|\delta|=22 \\
\delta=-2.3 \\
\xi=75.7 \\
\lambda=94.2\end{array}$ \\
\hline
\end{tabular}




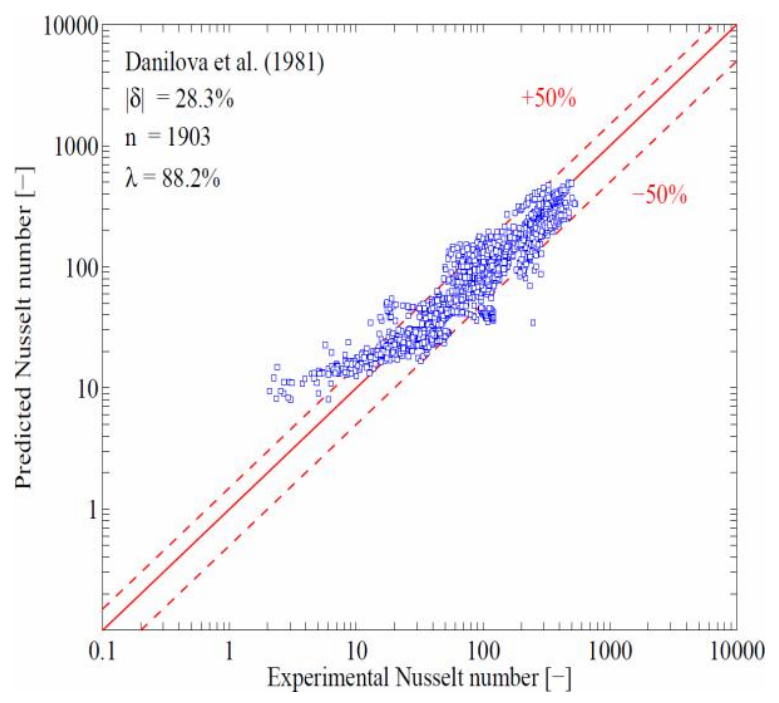

(a)

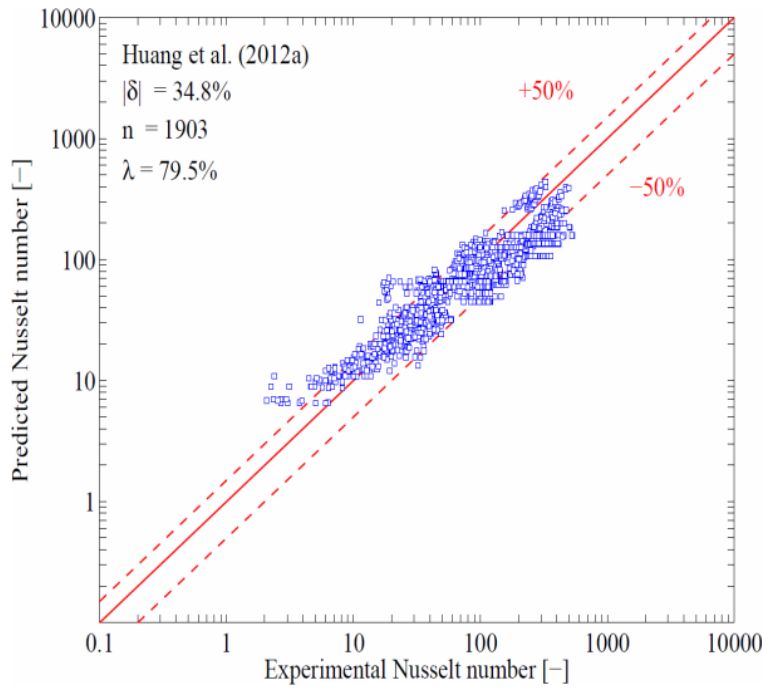

(c)

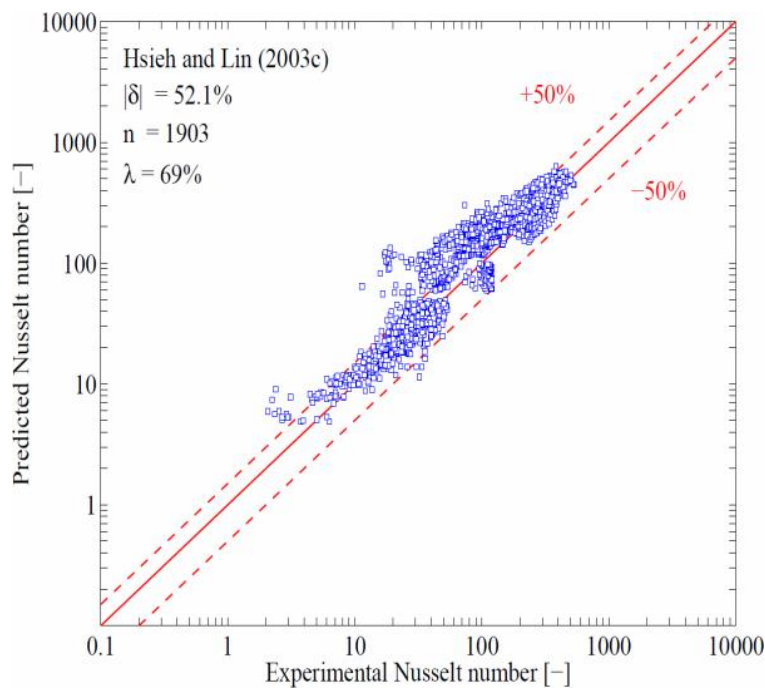

(e)

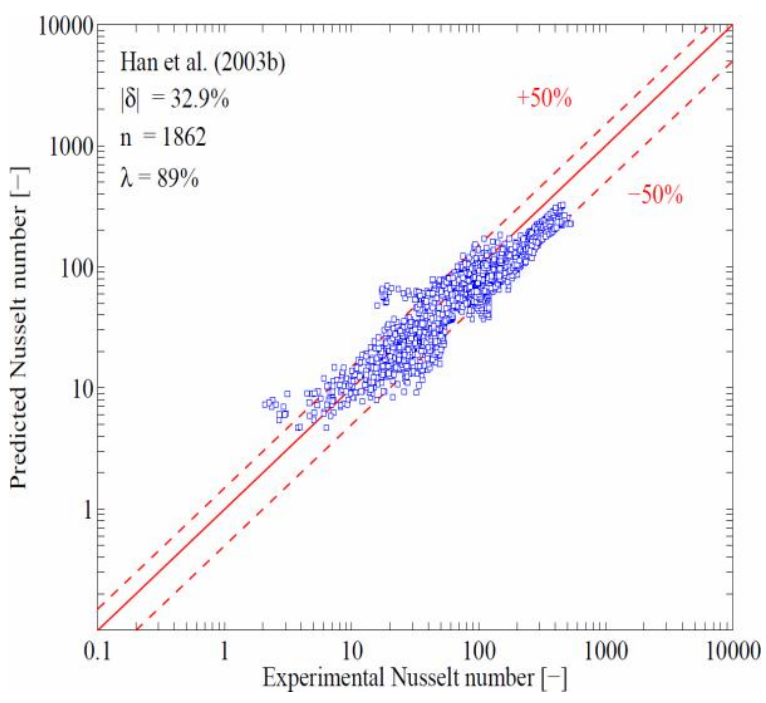

(b)

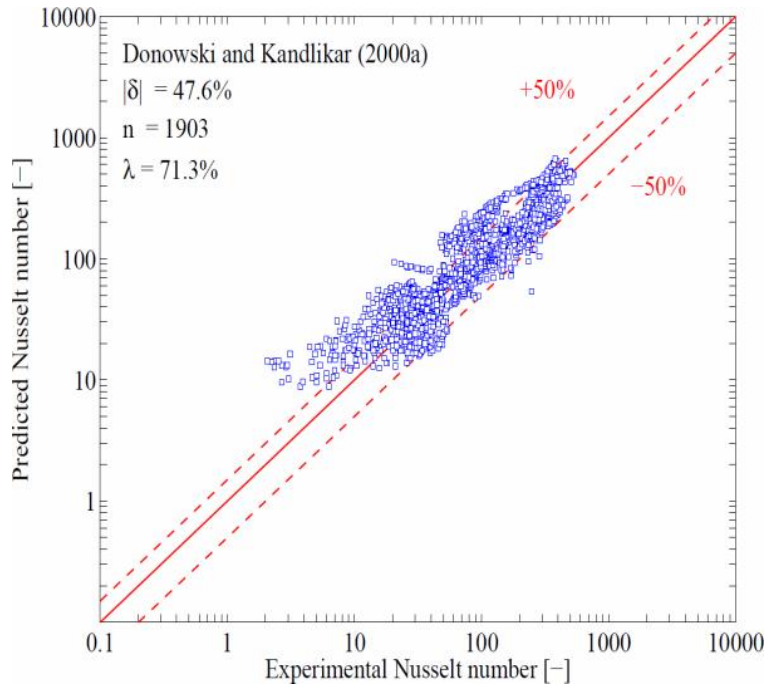

(d)

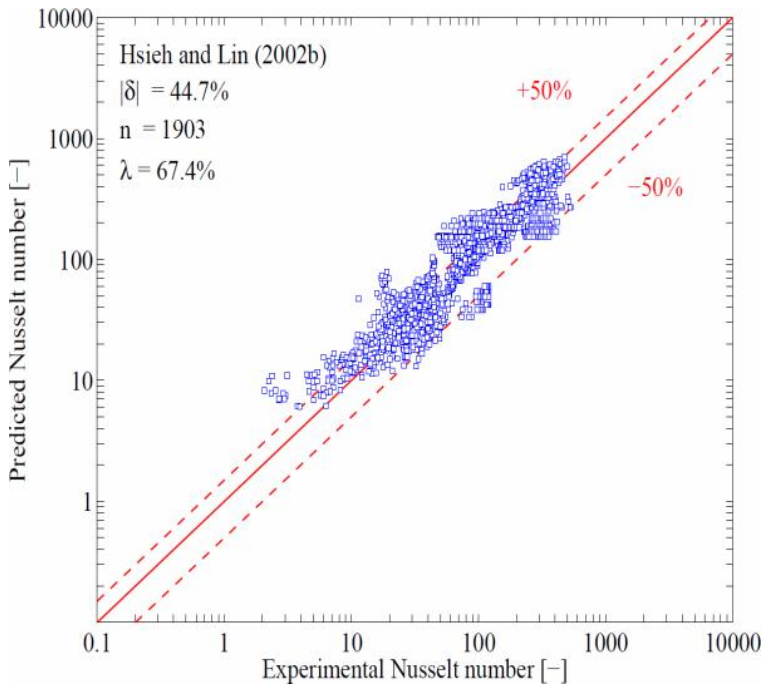

(f)

Fig. 6: Flow boiling predicted Nusselt number against experimental Nusselt number using the correlations proposed by: (a) Danilova et al. (1981), (b) Han et al. (2003b), (c) Huang et al. (2012a), (d) Donowski and Kandlikar (2000a), (e) and (f) Hsieh and Lin (2003c, 2002b). 
In Table 2 the predicted frictional pressure gradients are compared against the experimental database collected from the evaporation literature for plate heat exchangers (see Table 1, Part 1), using the $\pm 30 \%$ and $50 \%$ bandwidths for evaluating their prediction accuracy. As can be seen, all the methods are quite inconsistent, predicting only some of the other independent datasets well. This apparently means that either the way the data were reduced in the original experiment was questionable (such as leaving the inlet and outlet headers' pressure drops in the values reported for the plates) or more the geometric factors as well as the experimental parameters have not yet been accounted for well in the prediction methods.

The prediction methods of Lin and co-workers $(2003 \mathrm{c}, 2002 \mathrm{~b}, 1999)$ exhibited a moderate agreement to the data provided by the other independent studies, but could not predict the data of Huang et al. (2012a), Khan et al. (2014b, 2012b, 2012c), Lee et al. (2014a) and Vakili-Farahani et al. (2014c, 2014d). The Han et al. (2003b) method provided reasonable agreement with several independent experimental datasets, but it underpredicted the Lin data of (2003c, 2002b, 1999) and did not predict Khan et al. (2014b, 2012b, 2012c), Lee et al. (2014a) and Vakili-Farahani et al. (2014c, 2014d) data.

The Ayub (2003a) correlation underestimated most of the pressure drop results, but slightly overestimated the data of Park and Kim (2004) and Khan et al. (2014b, 2012b, 2012c), while it could not predict the results offered by Lee et al. (2014a). The Lee et al. (2014a) method exhibited poor predictive capabilities including their original data; in fact, only $13.9 \%$ of the pressure data were predicted within $\pm 50 \%$ with a mean absolute error of $51.6 \%$. Sterner and Sunden (2006c) suggested a prediction method based on the separated flow model approach, testing different PHEs. They correlated the frictional pressure drop considering that the $C$ factor changes with the liquid-only Reynolds number, which accounts for the effect mass velocity as well as the hydraulic diameter; however, this method still could not deal with the diverse datasets here: Huang et al. (2012a), Lee et al. (2014a), Longo et al. (2012d, 2012e, 2007a, 2007b, 2007c) and Vakili-Farahani et al. (2014c, 2014d). The Nilpueng and Wongwises (2010a) and Taboas et al. (2012f) methods underpredicted nearly the entire pressure drop databank. The Park and Kim (2004) method strongly overestimated most of the independent datasets, while it slightly underestimated the data provided by Lin et al. (2003c, 2002b, 1999) and Taboas et al. (2012f). Finally, the Huang et al. (2012a) method was seen to appropriately predict most of the databank; nevertheless, it could not predict the experimental data of Khan et al. (2014b, 2012b, 2012c). Similar results were observed using the Khan et al. (2014b, 2012c) methods. Fig. 7 depicts the evaporation frictional pressure drop databank (see Table 1, Part 1) compared to the corresponding values predicted by six selected prediction methods. In order to perform the comparison, the results are graphically presented and the mean absolute error, number of data points and the percentage of the point predicted within $\pm 50 \%$ are also reported. 
Table 2: Two-phase frictional pressure gradient predicted by the correlation along the rows compared to the individual experimental database along the columns.

\begin{tabular}{|c|c|c|c|c|c|c|c|c|c|}
\hline Author(s) & $\begin{array}{l}\text { Lin et al. } \\
(2002 a, 2003 c \text {, } \\
\text { 2002b, 1999) }\end{array}$ & $\begin{array}{l}\text { Taboas et al. } \\
(2012 \mathrm{f}, \\
2010 \mathrm{~b})\end{array}$ & $\begin{array}{l}\text { Huang et al. } \\
(2012 a)\end{array}$ & $\begin{array}{l}\text { Khan et al. } \\
(2014 b, 2012 b \text {, } \\
2012 c)\end{array}$ & $\begin{array}{l}\text { Lee et al. } \\
\text { (2014a) }\end{array}$ & $\begin{array}{l}\text { Longo et al. } \\
(2012 \mathrm{~d}, 2012 \mathrm{e}, 2007 \mathrm{a}, \\
2007 \mathrm{~b}, 2007 \mathrm{c})\end{array}$ & $\begin{array}{l}\text { Han et al. } \\
(2003 b)\end{array}$ & $\begin{array}{l}\text { Park and Kim } \\
\text { (2004) }\end{array}$ & $\begin{array}{l}\text { Vakili-Farahani et al. } \\
(2014 c, 2014 d)\end{array}$ \\
\hline $\begin{array}{l}\text { Yan and Lin } \\
\text { (1999) }\end{array}$ & $\begin{array}{l}|\delta|=\mathbf{4 1} \\
\delta=\mathbf{4 1} \\
\xi=\mathbf{2 7 . 7} \\
\lambda=\mathbf{5 9 . 3}\end{array}$ & $\begin{array}{l}|\delta|=27.8 \\
\delta=10.5 \\
\xi=61.5 \\
\lambda=80\end{array}$ & $\begin{array}{l}|\delta|=199 \\
\delta=199 \\
\xi=0 \\
\lambda=0\end{array}$ & $\begin{array}{l}|\delta|=1879.2 \\
\delta=1879.2 \\
\xi=0 \\
\lambda=0\end{array}$ & $\begin{array}{l}|\delta|=1923 \\
\delta=1923 \\
\xi=0 \\
\lambda=0\end{array}$ & $\begin{array}{l}|\delta|=104.6 \\
\delta=91.4 \\
\xi=24.5 \\
\lambda=39.5\end{array}$ & $\begin{array}{l}|\delta|=120.6 \\
\delta=120.6 \\
\xi=0 \\
\lambda=2\end{array}$ & $\begin{array}{l}|\delta|=96.5 \\
\delta=96.5 \\
\xi=6.2 \\
\lambda=11.5\end{array}$ & $\begin{array}{l}|\delta|=5282.1 \\
\delta=5282.1 \\
\xi=0 \\
\lambda=0\end{array}$ \\
\hline $\begin{array}{l}\text { Hsieh and Lin } \\
(2002 b)\end{array}$ & $\begin{array}{l}|\delta|=36.9 \\
\delta=\mathbf{3 6 . 9} \\
\xi=43.1 \\
\lambda=64.8\end{array}$ & $\begin{array}{l}|\delta|=55.2 \\
\delta=51.5 \\
\xi=35.4 \\
\lambda=44.6\end{array}$ & $\begin{array}{l}|\delta|=107.7 \\
\delta=107.7 \\
\xi=0 \\
\lambda=0\end{array}$ & $\begin{array}{l}|\delta|=823.9 \\
\delta=823.9 \\
\xi=0 \\
\lambda=0\end{array}$ & $\begin{array}{l}|\delta|=908.2 \\
\delta=908.2 \\
\xi=0 \\
\lambda=0\end{array}$ & $\begin{array}{l}|\delta|=31.4 \\
\delta=2.8 \\
\xi=49.1 \\
\lambda=78.9\end{array}$ & $\begin{array}{l}|\delta|=73 \\
\delta=73 \\
\xi=17.6 \\
\lambda=27.5\end{array}$ & $\begin{array}{l}|\delta|=54.2 \\
\delta=54.1 \\
\xi=23.9 \\
\lambda=46\end{array}$ & $\begin{array}{l}|\delta|=2213.9 \\
\delta=2213.9 \\
\xi=0 \\
\lambda=0\end{array}$ \\
\hline $\begin{array}{l}\text { Hsieh and Lin } \\
(2003 c)\end{array}$ & $\begin{array}{l}|\delta|=\mathbf{2 6 . 8} \\
\boldsymbol{\delta}=\mathbf{- 2 4 . 7} \\
\xi=\mathbf{5 0 . 6} \\
\lambda=\mathbf{8 8 . 3}\end{array}$ & $\begin{array}{l}|\delta|=79.5 \\
\delta=79.5 \\
\xi=33.8 \\
\lambda=35.4\end{array}$ & $\begin{array}{l}|\delta|=101.3 \\
\delta=101.3 \\
\xi=0 \\
\lambda=0\end{array}$ & $\begin{array}{l}|\delta|=800 \\
\delta=800 \\
\xi=0 \\
\lambda=0\end{array}$ & $\begin{array}{l}|\delta|=997 \\
\delta=997 \\
\xi=0 \\
\lambda=0\end{array}$ & $\begin{array}{l}|\delta|=28.6 \\
\delta=5.3 \\
\xi=56.1 \\
\lambda=86\end{array}$ & $\begin{array}{l}|\delta|=83.7 \\
\delta=83.7 \\
\xi=5.9 \\
\lambda=17.6\end{array}$ & $\begin{array}{l}|\delta|=84.2 \\
\delta=84.2 \\
\xi=3.6 \\
\lambda=20.4\end{array}$ & $\begin{array}{l}|\delta|=1759.9 \\
\delta=1759.9 \\
\xi=0 \\
\lambda=0\end{array}$ \\
\hline $\begin{array}{l}\text { Sterner and } \\
\text { Sunden } \\
(2006 \mathrm{c})\end{array}$ & $\begin{array}{l}|\delta|=55.4 \\
\delta=-26.6 \\
\xi=15.1 \\
\lambda=44.3\end{array}$ & $\begin{array}{l}|\delta|=196.8 \\
\delta=196.8 \\
\xi=0 \\
\lambda=0\end{array}$ & $\begin{array}{l}|\delta|=55.2 \\
\delta=-55.2 \\
\xi=0 \\
\lambda=16\end{array}$ & $\begin{array}{l}|\delta|=170.5 \\
\delta=165.6 \\
\xi=10.9 \\
\lambda=26.1\end{array}$ & $\begin{array}{l}|\delta|=493.4 \\
\delta=493.4 \\
\xi=0 \\
\lambda=0\end{array}$ & $\begin{array}{l}|\delta|=79.2 \\
\delta=-79.2 \\
\xi=0 \\
\lambda=0\end{array}$ & $\begin{array}{l}|\delta|=52.4 \\
\delta=-52.4 \\
\xi=7.8 \\
\lambda=41.2\end{array}$ & $\begin{array}{l}|\delta|=22.7 \\
\delta=-0.3 \\
\xi=69.9 \\
\lambda=92\end{array}$ & $\begin{array}{l}|\delta|=382.2 \\
\delta=379.4 \\
\xi=4.8 \\
\lambda=8\end{array}$ \\
\hline $\begin{array}{l}\text { Han et al. } \\
\text { (2003b) }\end{array}$ & $\begin{array}{l}|\delta|=43.8 \\
\delta=-43.8 \\
\xi=19.6 \\
\lambda=63.9\end{array}$ & $\begin{array}{l}|\delta|=19.4 \\
\delta=2.1 \\
\xi=84.6 \\
\lambda=100\end{array}$ & $\begin{array}{l}|\delta|=20.8 \\
\delta=-20.8 \\
\xi=90 \\
\lambda=100\end{array}$ & $\begin{array}{l}|\delta|=430.5 \\
\delta=430.5 \\
\xi=0 \\
\lambda=0\end{array}$ & $\begin{array}{l}|\delta|=433.1 \\
\delta=433.1 \\
\xi=0 \\
\lambda=0\end{array}$ & $\begin{array}{l}|\delta|=47.5 \\
\delta=-47.5 \\
\xi=6.1 \\
\lambda=55.3\end{array}$ & $\begin{array}{l}|\delta|=18.1 \\
\delta=17.9 \\
\xi=90.2 \\
\lambda=96.1\end{array}$ & $\begin{array}{l}|\delta|=58.4 \\
\delta=-58.4 \\
\xi=0.3 \\
\lambda=28.3\end{array}$ & $\begin{array}{l}|\delta|=548 \\
\delta=548 \\
\xi=0.5 \\
\lambda=0.8\end{array}$ \\
\hline $\begin{array}{l}\text { Ayub } \\
(2003 a)\end{array}$ & $\begin{array}{l}|\delta|=48.1 \\
\delta=-47.8 \\
\xi=25 \\
\lambda=42.8\end{array}$ & $\begin{array}{l}|\delta|=19.9 \\
\delta=-0.8 \\
\xi=81.6 \\
\lambda=100\end{array}$ & $\begin{array}{l}|\delta|=75.6 \\
\delta=-75.6 \\
\xi=0 \\
\lambda=0\end{array}$ & $\begin{array}{l}|\delta|=69.4 \\
\delta=44.4 \\
\xi=35.9 \\
\lambda=55.4\end{array}$ & $\begin{array}{l}|\delta|=354.3 \\
\delta=354.3 \\
\xi=0 \\
\lambda=0\end{array}$ & $\begin{array}{l}|\delta|=82.6 \\
\delta=-82.6 \\
\xi=0 \\
\lambda=0\end{array}$ & $\begin{array}{l}|\delta|=52.3 \\
\delta=-52.3 \\
\xi=21.6 \\
\lambda=49\end{array}$ & $\begin{array}{l}|\delta|=52.7 \\
\delta=26.9 \\
\xi=26.5 \\
\lambda=44.2\end{array}$ & $\begin{array}{l}|\delta|=53.1 \\
\delta=-53.1 \\
\xi=8.2 \\
\lambda=47.3\end{array}$ \\
\hline $\begin{array}{l}\text { Nilpueng and } \\
\text { Wongwises } \\
\text { (2010a) }\end{array}$ & $\begin{array}{l}|\delta|=73.7 \\
\delta=-73.7 \\
\xi=0 \\
\lambda=5.4\end{array}$ & $\begin{array}{l}|\delta|=36.2 \\
\delta=-36.2 \\
\xi=10.8 \\
\lambda=100\end{array}$ & $\begin{array}{l}|\delta|=86 \\
\delta=-86 \\
\xi=0 \\
\lambda=0\end{array}$ & $\begin{array}{l}|\delta|=73.4 \\
\delta=-73.4 \\
\xi=5.4 \\
\lambda=16.3\end{array}$ & $\begin{array}{l}|\delta|=62.2 \\
\delta=-62.2 \\
\xi=4.2 \\
\lambda=23.6\end{array}$ & $\begin{array}{l}|\delta|=94.7 \\
\delta=-94.7 \\
\xi=0 \\
\lambda=0\end{array}$ & $\begin{array}{l}|\delta|=83.8 \\
\delta=-83.8 \\
\xi=0 \\
\lambda=0\end{array}$ & $\begin{array}{l}|\delta|=70.4 \\
\delta=-70.4 \\
\xi=0 \\
\lambda=6.2\end{array}$ & $\begin{array}{l}|\delta|=71.5 \\
\delta=-71.5 \\
\xi=0 \\
\lambda=4\end{array}$ \\
\hline $\begin{array}{l}\text { Jokar et al. } \\
\text { (2006a) }\end{array}$ & $\begin{array}{l}|\delta|=70.4 \\
\delta=-70.4 \\
\xi=0.9 \\
\lambda=13.6\end{array}$ & $\begin{array}{l}|\delta|=82.6 \\
\delta=-82.6 \\
\xi=0 \\
\lambda=0\end{array}$ & $\begin{array}{l}|\delta|=13.4 \\
\delta=-6.2 \\
\xi=94 \\
\lambda=100\end{array}$ & $\begin{array}{l}|\delta|=437.9 \\
\delta=437.9 \\
\xi=0 \\
\lambda=0\end{array}$ & $\begin{array}{l}|\delta|=510.1 \\
\delta=510.1 \\
\xi=0 \\
\lambda=0\end{array}$ & $\begin{array}{l}|\delta|=51.7 \\
\delta=-51.4 \\
\xi=18.4 \\
\lambda=40.4\end{array}$ & $\begin{array}{l}|\delta|=29.8 \\
\delta=-29 \\
\xi=56.9 \\
\lambda=88.2\end{array}$ & $\begin{array}{l}|\delta|=32.8 \\
\delta=-32.8 \\
\xi=46 \\
\lambda=84.1\end{array}$ & $\begin{array}{l}|\delta|=1783.3 \\
\delta=1783.3 \\
\xi=0.2 \\
\lambda=0.6\end{array}$ \\
\hline $\begin{array}{l}\text { Khan et al. } \\
(2014 b, \\
2012 b, 2012 c)\end{array}$ & $\begin{array}{l}|\delta|=39.6 \\
\delta=-39.6 \\
\xi=29.8 \\
\lambda=73.2\end{array}$ & $\begin{array}{l}|\delta|=15.3 \\
\delta=1.8 \\
\xi=93.9 \\
\lambda=100\end{array}$ & $\begin{array}{l}|\delta|=143.4 \\
\delta=143.4 \\
\xi=0 \\
\lambda=0\end{array}$ & $\begin{array}{l}|\delta|=\mathbf{5 1 . 2} \\
\delta=\mathbf{1 0 . 3} \\
\xi=\mathbf{1 8 . 5} \\
\lambda=\mathbf{6 9 . 6}\end{array}$ & $\begin{array}{l}|\delta|=33.7 \\
\delta=-22.3 \\
\xi=44.4 \\
\lambda=73.6\end{array}$ & $\begin{array}{l}|\delta|=70.7 \\
\delta=-70.7 \\
\xi=0 \\
\lambda=0\end{array}$ & $\begin{array}{l}|\delta|=65.3 \\
\delta=33.2 \\
\xi=54.9 \\
\lambda=64.7\end{array}$ & $\begin{array}{l}|\delta|=17.2 \\
\delta=-13.4 \\
\xi=80.5 \\
\lambda=99.1\end{array}$ & $\begin{array}{l}|\delta|=24.8 \\
\delta=1.9 \\
\xi=66.3 \\
\lambda=93.1\end{array}$ \\
\hline
\end{tabular}


Table 2: Two-phase frictional pressure gradient predicted by the correlation along the rows compared to the individual experimental database along the columns (continued).

\begin{tabular}{|c|c|c|c|c|c|c|c|c|c|}
\hline Author(s) & $\begin{array}{l}\text { Lin et al. } \\
(2002 a, 2003 c \text {, } \\
\text { 2002b, 1999) }\end{array}$ & $\begin{array}{l}\text { Taboas et al. } \\
(2012 \mathrm{f} \text {, } \\
2010 \mathrm{~b})\end{array}$ & $\begin{array}{l}\text { Huang et al. } \\
(2012 a)\end{array}$ & $\begin{array}{l}\text { Khan et al. } \\
(2014 b, 2012 b, \\
2012 c)\end{array}$ & $\begin{array}{l}\text { Lee et al. } \\
\text { (2014a) }\end{array}$ & $\begin{array}{l}\text { Longo et al. } \\
(2012 \mathrm{~d}, 2012 \mathrm{e}, 2007 \mathrm{a} \text {, } \\
2007 \mathrm{~b}, 2007 \mathrm{c})\end{array}$ & $\begin{array}{l}\text { Han et al. } \\
\text { (2003b) }\end{array}$ & $\begin{array}{l}\text { Park and Kim } \\
(2004)\end{array}$ & $\begin{array}{l}\text { Vakili-Farahani et al. } \\
(2014 c, 2014 d)\end{array}$ \\
\hline $\begin{array}{l}\text { Taboas et al. } \\
(2012 \mathrm{f})\end{array}$ & $\begin{array}{l}|\delta|=36.2 \\
\delta=-35 \\
\xi=47.6 \\
\lambda=66\end{array}$ & $\begin{array}{l}|\delta|=15.5 \\
\delta=14.8 \\
\xi=92.3 \\
\lambda=100\end{array}$ & $\begin{array}{l}|\delta|=70.2 \\
\delta=-70.2 \\
\xi=0 \\
\lambda=0\end{array}$ & $\begin{array}{l}|\delta|=36.1 \\
\delta=-20.2 \\
\xi=47.8 \\
\lambda=72.8\end{array}$ & $\begin{array}{l}|\delta|=93.5 \\
\delta=93.5 \\
\xi=0 \\
\lambda=0\end{array}$ & $\begin{array}{l}|\delta|=77.5 \\
\delta=-77.5 \\
\xi=0 \\
\lambda=0\end{array}$ & $\begin{array}{l}|\delta|=43.4 \\
\delta=-43.4 \\
\xi=27.5 \\
\lambda=66.7\end{array}$ & $\begin{array}{l}|\delta|=50.3 \\
\delta=22.6 \\
\xi=27.4 \\
\lambda=45.1\end{array}$ & $\begin{array}{l}|\delta|=56 \\
\delta=-56 \\
\xi=2.7 \\
\lambda=41.8\end{array}$ \\
\hline $\begin{array}{l}\text { Huang et al. } \\
\text { (2012a) }\end{array}$ & $\begin{array}{l}|\delta|=35.4 \\
\delta=-27.2 \\
\xi=33.1 \\
\lambda=83.4\end{array}$ & $\begin{array}{l}|\delta|=40.9 \\
\delta=37.6 \\
\xi=35.4 \\
\lambda=55.4\end{array}$ & $\begin{array}{l}|\boldsymbol{\delta}|=\mathbf{9 . 9} \\
\boldsymbol{\delta}=\mathbf{- 8 . 7} \\
\xi=\mathbf{1 0 0} \\
\lambda=\mathbf{1 0 0}\end{array}$ & $\begin{array}{l}|\delta|=185 \\
\delta=185 \\
\xi=0 \\
\lambda=2.2\end{array}$ & $\begin{array}{l}|\delta|=412 \\
\delta=412 \\
\xi=0 \\
\lambda=0\end{array}$ & $\begin{array}{l}|\delta|=43.6 \\
\delta=-43.6 \\
\xi=10.6 \\
\lambda=70.2\end{array}$ & $\begin{array}{l}|\delta|=24.5 \\
\delta=24.5 \\
\xi=86.3 \\
\lambda=100\end{array}$ & $\begin{array}{l}|\delta|=14.2 \\
\delta=-10.6 \\
\xi=86.7 \\
\lambda=98.2\end{array}$ & $\begin{array}{l}|\delta|=307.9 \\
\delta=307.3 \\
\xi=0.8 \\
\lambda=1.6\end{array}$ \\
\hline $\begin{array}{l}\text { Park and Kim } \\
\text { (2004) }\end{array}$ & $\begin{array}{l}|\delta|=58.8 \\
\delta=-58.8 \\
\xi=2.1 \\
\lambda=30.4\end{array}$ & $\begin{array}{l}|\delta|=34.9 \\
\delta=-18.5 \\
\xi=50.8 \\
\lambda=78.5\end{array}$ & $\begin{array}{l}|\delta|=338.5 \\
\delta=338.5 \\
\xi=0 \\
\lambda=0\end{array}$ & $\begin{array}{l}|\delta|=2820.8 \\
\delta=2820.8 \\
\xi=0 \\
\lambda=0\end{array}$ & $\begin{array}{l}|\delta|=1887 \\
\delta=1887 \\
\xi=0 \\
\lambda=0\end{array}$ & $\begin{array}{l}|\delta|=156 \\
\delta=140.3 \\
\xi=19.3 \\
\lambda=30.7\end{array}$ & $\begin{array}{l}|\delta|=135.3 \\
\delta=135.3 \\
\xi=3.9 \\
\lambda=5.9\end{array}$ & $\begin{array}{l}|\delta|=39.4 \\
\delta=39.4 \\
\xi=28.3 \\
\lambda=79.6\end{array}$ & $\begin{array}{l}|\delta|=1612.2 \\
\delta=1612.2 \\
\xi=0 \\
\lambda=0\end{array}$ \\
\hline $\begin{array}{l}\text { Lee et al. } \\
\text { (2014a) }\end{array}$ & $\begin{array}{l}|\delta|=97.7 \\
\delta=-97.7 \\
\xi=0 \\
\lambda=0\end{array}$ & $\begin{array}{l}|\delta|=95.3 \\
\delta=-95.3 \\
\xi=0 \\
\lambda=0\end{array}$ & $\begin{array}{l}|\delta|=95.9 \\
\delta=-95.9 \\
\xi=0 \\
\lambda=0\end{array}$ & $\begin{array}{l}|\delta|=74.3 \\
\delta=-74.3 \\
\xi=0 \\
\lambda=4.4\end{array}$ & $\begin{array}{l}|\boldsymbol{\delta}|=\mathbf{5 1 . 6} \\
\boldsymbol{\delta}=\mathbf{- 5 0 . 9} \\
\xi=\mathbf{1 3 . 9} \\
\lambda=\mathbf{3 7 . 5}\end{array}$ & $\begin{array}{l}|\delta|=96.7 \\
\delta=-96.7 \\
\xi=0 \\
\lambda=0\end{array}$ & $\begin{array}{l}|\delta|=94.9 \\
\delta=-94.8 \\
\xi=0 \\
\lambda=0\end{array}$ & $\begin{array}{l}|\delta|=92.6 \\
\delta=-92.6 \\
\xi=0 \\
\lambda=0\end{array}$ & $\begin{array}{l}|\delta|=75.4 \\
\delta=-75.4 \\
\xi=0 \\
\lambda=0\end{array}$ \\
\hline $\begin{array}{l}\text { Present } \\
\text { method }\end{array}$ & $\begin{array}{l}|\delta|=19.2 \\
\delta=0.5 \\
\xi=80.7 \\
\lambda=95.8\end{array}$ & $\begin{array}{l}|\delta|=20.7 \\
\delta=-0.4 \\
\xi=66.2 \\
\lambda=100\end{array}$ & $\begin{array}{l}|\delta|=19.4 \\
\delta=-19.4 \\
\xi=90 \\
\lambda=100\end{array}$ & $\begin{array}{l}|\delta|=24.1 \\
\delta=-11.3 \\
\xi=63 \\
\lambda=95.7\end{array}$ & $\begin{array}{l}|\delta|=44.7 \\
\delta=-41.5 \\
\xi=23.6 \\
\lambda=52.8\end{array}$ & $\begin{array}{l}|\delta|=37.4 \\
\delta=-31 \\
\xi=35.1 \\
\lambda=65.8\end{array}$ & $\begin{array}{l}|\delta|=42.7 \\
\delta=41.6 \\
\xi=43.1 \\
\lambda=64.7\end{array}$ & $\begin{array}{l}|\delta|=26.1 \\
\delta=-25.5 \\
\xi=63.7 \\
\lambda=91.2\end{array}$ & $\begin{array}{l}|\delta|=14.4 \\
\delta=-1.9 \\
\xi=89.1 \\
\lambda=97.1\end{array}$ \\
\hline
\end{tabular}




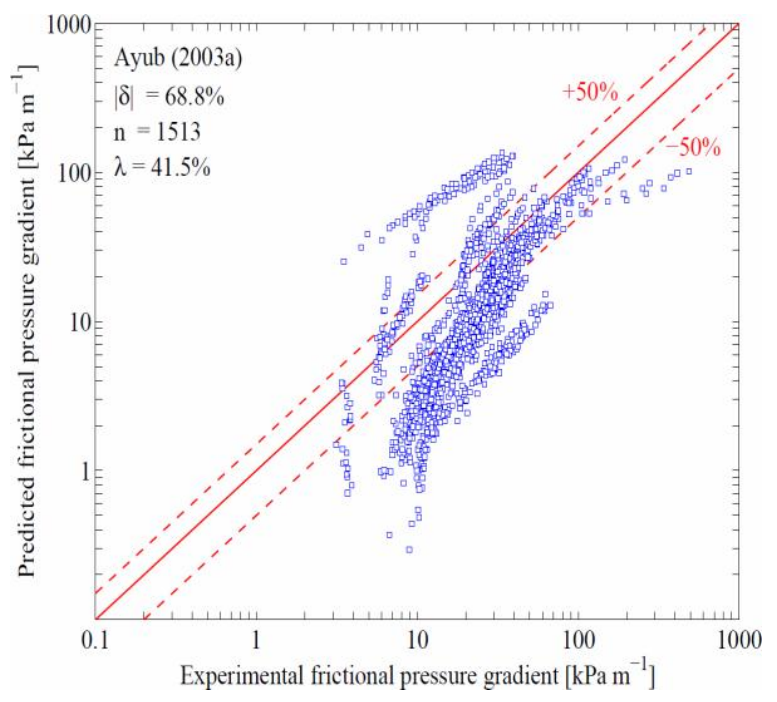

(a)

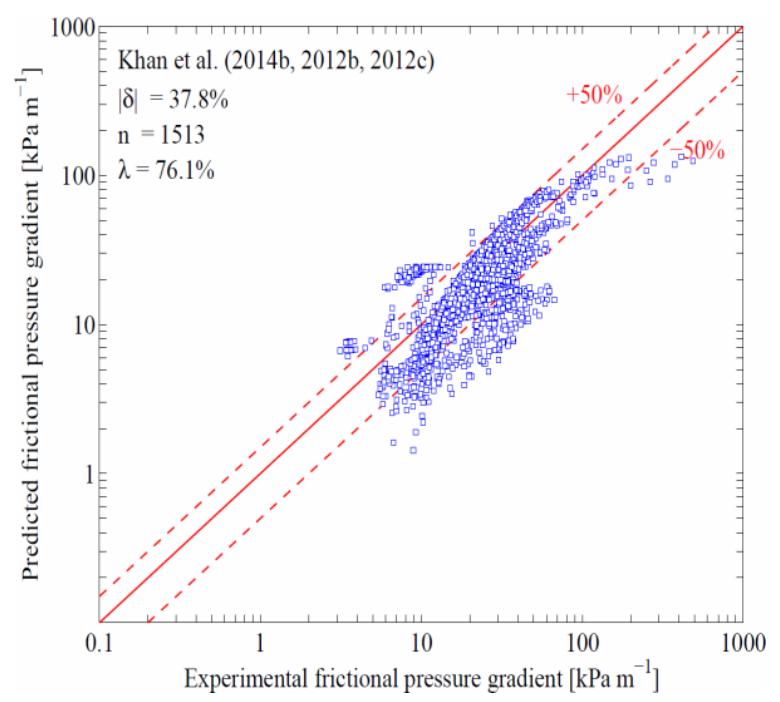

(c)

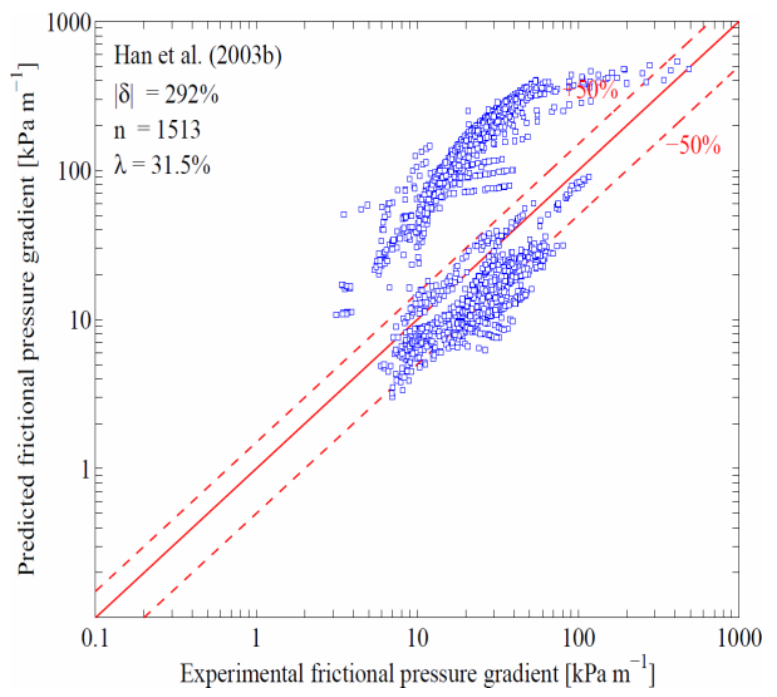

(e)

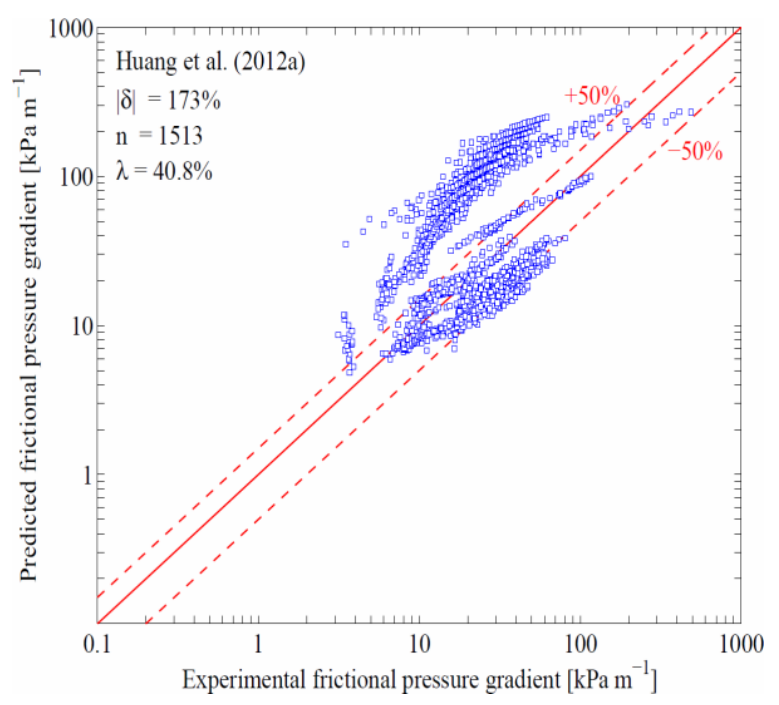

(b)

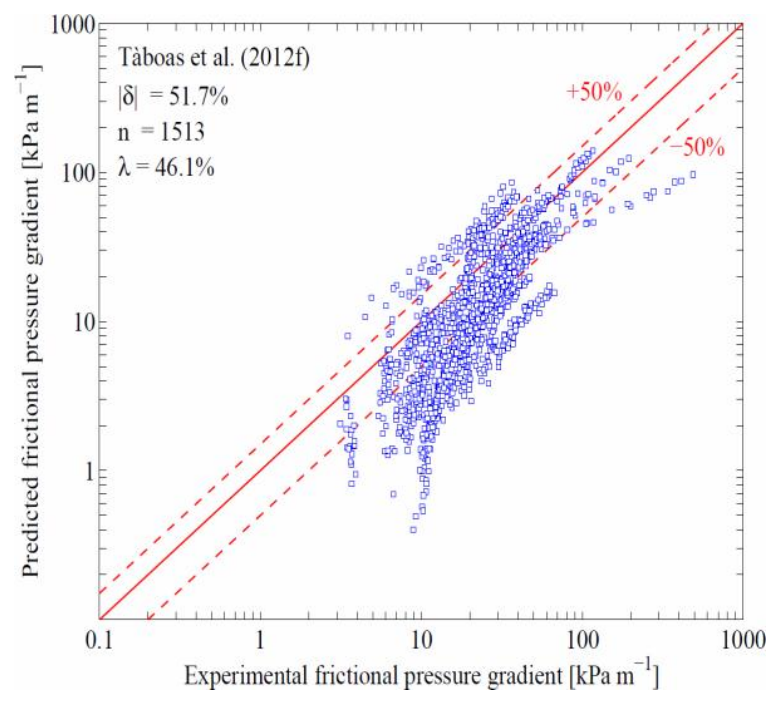

(d)

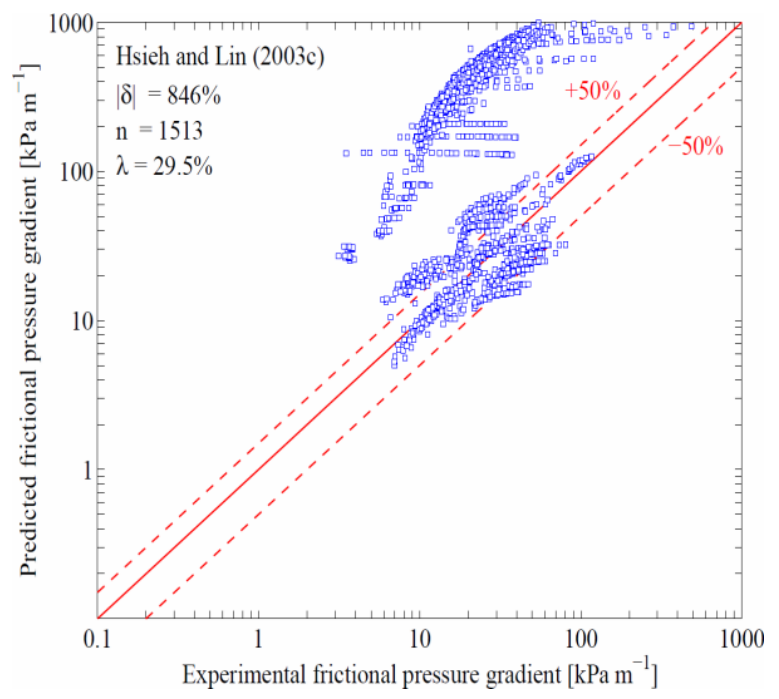

(f)

Fig. 7: Flow boiling predicted frictional pressure gradient against experimental pressure gradient using the correlations proposed by: (a) Ayub (2003a), (b) Huang et al. (2012a), (c) Khan et al. (2014b, 2012b, 2012c), (d) Taboas et al. (2012f), (a) Han et al. (2003b), (b) Hsieh and Lin (2003c). 


\section{Dimension analysis and new flow boiling heat transfer and pressure drop prediction methods}

In what follows, a new flow boiling approach is taken to predict frictional pressure gradients and heat transfer coefficients within plate heat exchangers. The technique adopted here has been previously used in some pool boiling and flow boiling studies, that it is based on dimensional analysis coupled with a multiple regression analysis, which identifies the most important parameters that can influence thermal and hydraulic performance.

In order to develop a prediction method for the local frictional pressure gradient, the wall shear stress was assumed to be dependent on the chevron angle $\beta$, hydraulic diameter $d_{h}$, densities of the liquid $\rho_{1}$ and vapor phases $\rho_{v}$, homogeneous density $\rho_{\mathrm{m}}$, superficial velocities of the liquid $\mathrm{u}_{\mathrm{l}}$ and vapor phases $\mathrm{u}_{\mathrm{v}}$, homogeneous superficial velocity $\rho_{\mathrm{m}}$, surface tension $\sigma$, dynamic viscosities of the liquid $\mu_{1}$ and vapor phases $\mu_{v}$ and the gravitational term $\left(\rho_{1}-\rho_{v}\right) g$ to consider the buoyancy force. Consequently, the shear stress was expressed with the following independent variables:

$$
\tau_{\text {wall }}=\tau_{\text {wall }}\left(\beta, d_{h}, \rho_{l}, \rho_{v}, \rho_{m}, u_{l}, u_{v}, u_{m}, \sigma, \mu_{l}, \mu_{v},\left(\rho_{l}-\rho_{v}\right) g\right)
$$

Next, the homogeneous density, velocity and hydraulic diameter were selected to take into account the primary dimensions involved:

$$
[M]=\rho_{m} d_{h}^{3}, \quad[L]=d_{h}, \quad[t]=u_{m}^{-1} d_{h}
$$

As a consequence, the number of dimensionless parameters to be included in this analysis was found to be equal to nine. The variables mentioned in eq. 2 were integrated with each variable introduced in eq. 1 to determine the non-dimensional parameters. Based on dimensional analysis, eq. 1 can be express in dimensionless form as follows:

$$
\begin{gathered}
f_{t p}=f_{t p}\left(\Pi_{1}, \Pi_{2}, \ldots \ldots ., \Pi_{9}\right) \\
\Pi_{1}=\frac{\sigma}{\rho_{m} u_{m}^{2} d_{h}}, \quad \Pi_{2}=\frac{\mu_{l}}{\rho_{m} u_{m} d_{h}}, \quad \Pi_{3}=\frac{\mu_{v}}{\rho_{m} u_{m} d_{h}}, \quad \Pi_{4}=\frac{\rho_{l}}{\rho_{m}} \\
\Pi_{5}=\frac{\rho_{v}}{\rho_{m}}, \quad \Pi_{6}=\beta, \quad \Pi_{7}=\frac{u_{l}}{u_{m}}, \quad \Pi_{8}=\frac{u_{v}}{u_{m}}, \quad \Pi_{9}=\frac{\left(\rho_{l}-\rho_{v}\right) g d_{h}}{\rho_{m} u_{m}^{2}}
\end{gathered}
$$

The non-dimensional groups were then manipulated in order to simplify their interpretations and attempt to capture the physical mechanisms involved in the two-phase flow process:

$$
\begin{aligned}
& \Psi_{1}=\Pi_{1}^{-1}=\frac{\rho_{m} u_{m}^{2} d_{h}}{\sigma}=\frac{G^{2} d_{h}}{\rho_{m} \sigma}=W e_{m} \\
& \Psi_{2}=\Pi_{2}^{-1}=\frac{\rho_{m} u_{m} d_{h}}{\mu_{l}}=\frac{G d_{h}}{\mu_{l}}=\operatorname{Re}_{l o} \\
& \Psi_{3}=\Pi_{3}^{-1}=\frac{\rho_{m} u_{m} d_{h}}{\mu_{v}}=\frac{G d_{h}}{\mu_{v}}=\operatorname{Re}_{v o} \\
& \Psi_{4}=\Pi_{4} \cdot \Pi_{5}^{-1}=\frac{\rho_{l}}{\rho_{v}}=\rho^{*} \\
& \Psi_{5}=\Pi_{6}=\frac{\beta}{\beta_{\max }}=\beta^{*} \\
& \Psi_{6}=\Pi_{7} \cdot \Pi_{4} \cdot \Pi_{2}^{-1}=\frac{G(1-x) d_{h}}{\mu_{l}}=\operatorname{Re}_{l} \\
& \Psi_{7}=\Pi_{8} \cdot \Pi_{5} \cdot \Pi_{3}^{-1}=\frac{G x d_{h}}{\mu_{v}}=\operatorname{Re}_{v} \\
& \Psi_{8}=\Pi_{9} \cdot \Pi_{1}^{-1}=\frac{\left(\rho_{l}-\rho_{v}\right) g d_{h}^{2}}{\sigma}=B d
\end{aligned}
$$


From the above manipulations, some well-known dimensionless groups were obtained, which are commonly used to provide some physical insight of the two-phase flow mechanisms. The homogeneous Weber number $\mathrm{We}_{\mathrm{m}}$ is the ratio between the inertia and tension forces and is adopted to characterize flow regime transitions in some flow pattern maps. The liquid only and vapor only Reynolds numbers, $\mathrm{Re}_{\mathrm{lo}}$ and $\mathrm{Re}_{\mathrm{vo}}$, are ratios between inertial to viscous forces and they consider respectively that the flow streaming in the plate is totally liquid or vapor. The non-dimensional groups $\Psi_{4}$ and $\Psi_{5}$ were combined to obtain the ratio between the liquid and vapor densities $\rho^{*}$. This parameter is adopted here to take into account the fluid properties, which can significantly change from one fluid to another. The dimensionless group $\Psi_{6}$ took into consideration the effect of the chevron angle $\beta$ (see Part 1 for the definition), which is one of the most important geometrical parameter in PHEs and it can strongly affect the performance as well as the flow distribution. In the present analysis $\beta_{\max }$ was the largest chevron angle investigated in the current databank and it was equal to $70^{\circ}$. The liquid and vapor Reynolds numbers, $R_{\mathrm{I}}$ and $\mathrm{Re}_{\mathrm{v}}$, are commonly used in the separated flow model approach, in which it is important to distinguish between liquid and vapor phase contributions on the flow boiling mechanism. The Bond number $\mathrm{Bd}$ represents the ratio between the buoyancy and the surface tension forces and it has been used in recent years to characterize the transition between macro-to-microscale two-phase flows as the plate's corrugation height is decreased. Finally, their ranges in terms of the present database are listed in the Table 3.

Table 3: Range of dimensionless numbers for flow boiling pressure drop database.

\begin{tabular}{|c|c|c|}
\hline Parameter & Min value & Max value \\
\hline Fanning friction factor, $\mathrm{f}_{\mathrm{tp}}$ & $1.75 \cdot 10^{-1}$ & $3.57 \cdot 10^{1}$ \\
\hline Homogeneous Weber number, $\mathrm{We}_{\mathrm{m}}$ & $2.67 \cdot 10^{-2}$ & $1.50 \cdot 10^{2}$ \\
\hline Liquid only Reynolds number, $\operatorname{Re}_{l o}$ & $3.31 \cdot 10^{1}$ & $4.74 \cdot 10^{3}$ \\
\hline Vapor only Reynolds number, $\operatorname{Re}_{\mathrm{vo}}$ & $1.69 \cdot 10^{3}$ & $5.98 \cdot 10^{4}$ \\
\hline Density ratio, $\rho^{*}$ & $1.91 \cdot 10^{1}$ & $1.35 \cdot 10^{3}$ \\
\hline Reduced chevron angle, $\beta^{*}$ & $4.29 \cdot 10^{-1}$ & $9.29 \cdot 10^{-1}$ \\
\hline Liquid Reynolds number, $\mathrm{Re}_{\mathrm{l}}$ & 8.02 & $4.72 \cdot 10^{3}$ \\
\hline Vapor Reynolds number, $\operatorname{Re}_{\mathrm{v}}$ & $1.01 \cdot 10^{1}$ & $3.46 \cdot 10^{4}$ \\
\hline Bond number, Bd & 2.40 & $4.91 \cdot 10^{1}$ \\
\hline
\end{tabular}

The two-phase Fanning friction factor was correlated based on the dimensionless numbers mentioned in the eq. 5 by adopting the power law representation as shown below:

$$
f_{t p}=f_{t p}\left(\Psi_{i}\right)=C \cdot \Psi_{i}^{a_{i}}, \quad i=1,2, \ldots \ldots, 8
$$

For the present investigation, the power law scheme coupled with a multiple regression analysis was adopted to calculate the leading coefficient $\mathrm{C}$ and the exponents $\mathrm{a}_{\mathrm{i}}$ which return the best fit of the entire experimental database. Precisely, for each dimensionless group the leading constant and exponents were evaluated by fitting the experimental databank based using a least square method, where the smallest square error sum identifies the most influential groups. Finally, this procedure was applied until all the dimensionless numbers were grouped. Even though all dimensionless numbers could be kept for correlating the two-phase Fanning friction factor, it is common practice to define a ranking of importance, thus neglecting the non-dimensional numbers that provide little gain in accuracy and thus simplifying the resulting correlation. In addition, a prediction method which is easy to implement and able to predict the flow boiling performance over a wide range of test conditions is preferred.

According to the experimental database, the two-phase Fanning friction was best correlated based on the homogeneous Weber number, Bond number, density ratio (liquid/vapor) and chevron angle. Here, it is interesting to note that the annular flow model of Cioncolini et al. (2009) found the core Weber number to be the most dominant group in their similar analysis for tubular annular flows. The other non-dimensional numbers (liquid/liquid only and vapor/vapor only Reynolds numbers) were eliminated here because they did not provide any improvements for the prediction 
capability. Finally, the two-phase Fanning friction factor and thus the frictional pressure gradient within PHE's can be predicted as follows:

$$
f_{t p}=C \cdot 15.698 \cdot W e_{m}^{-0.475} B d^{0.255} \rho^{*-0.571}
$$

where the $\mathrm{C}$ parameter takes into consideration the effect of the corrugation angle (expressed in degrees, not radians) on the pressure drop and it was correlated as:

$$
C=2.125 \beta^{* 9.993}+0.955
$$

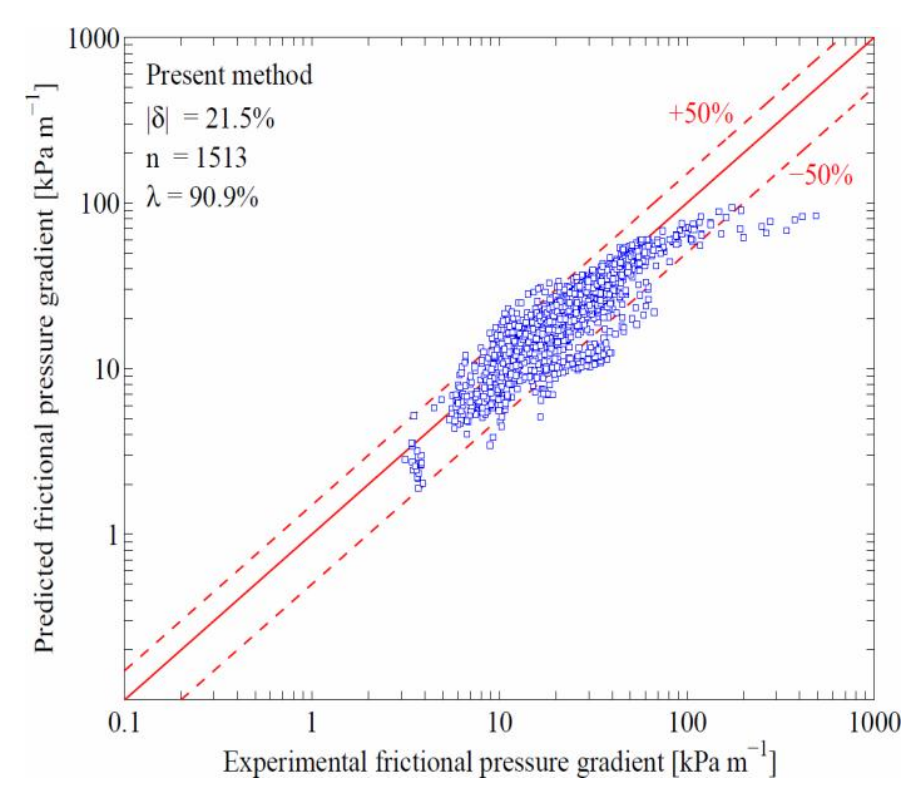

Fig. 8: Prediction method for two-phase frictional pressure drops within PHEs: comparison against experimental frictional pressure gradient.

In Fig. 8, the comparisons between the predicted and experimental frictional pressure gradient are shown and a reasonable agreement with the experimental database was found. Precisely, the proposed method is able to predict $90.9 \%$ of the experimental frictional pressure drop database (which included 1513 data points) with a mean absolute error of $21.5 \%$, mean error of $-6.8 \%$, within a bandwidth of $\pm 50 \%$ while $74.1 \%$ of the data fall within $\pm 30 \%$. With respect to Fig. 7, there is a visible significant improvement.

One can see that the friction factor increases with chevron angle because of the longer fluid path, but decreases with the Weber number. The reason may be explained by the fact that with an increasing vapor quality the liquid film thickness close to the wall becomes thinner and as a consequence lower shear stress can be observed. Furthermore, the friction factor rises when the Bond number grows because of the late transition from laminar to turbulent flow regime, which provides higher shear stress but on the other hand it decreases with an increase of the density ratio.

To develop a more general prediction method for flow boiling heat transfer within PHEs, the same procedure as above was followed. In the case of the heat transfer coefficient, 3 more independent variables were added: the latent heat of vaporization $\mathrm{i}_{\mathrm{lv}}$, the heat flux $\mathrm{q}$, and the thermal conductivity of the liquid phase $\mathrm{k}_{\mathrm{l}}$. The final relationship is as follows:

$$
h_{t p}=h_{t p}\left(\beta, d_{h}, \rho_{l}, \rho_{v}, \rho_{m}, u_{l}, u_{v}, u_{m}, \sigma, \mu_{l}, \mu_{v},\left(\rho_{l}-\rho_{v}\right) g, i_{l v}, q, k_{l}\right)
$$

In addition to the homogeneous density, velocity and hydraulic diameter, the liquid thermal conductivity was also selected to be taken into consideration to include the effect of temperature as a primary dimension, which was not included in the pressure drop analysis, and thus:

$$
[M]=\rho_{m} d_{h}^{3}, \quad[L]=d_{h}, \quad[t]=u_{m}^{-1} d_{h}, \quad[T]=\rho_{m} d_{h} u_{m}^{-3} k_{l}
$$


As a consequence, the number of dimensionless groups is 11 . Using the parameters selected in eq.10, according to the dimensional analysis, eq. 9 can be express in dimensionless form as follows:

$$
N u_{t p}=N u_{t p}\left(\Pi_{1}, \Pi_{2}, \ldots \ldots, \Pi_{11}\right)
$$

and two extra dimensionless numbers appeared:

$$
\Pi_{10}=\frac{i_{l v}}{u_{m}^{2}}, \quad \Pi_{11}=\frac{q}{\rho_{m} u_{m}^{3}}
$$

Next, the above two parameters were combined to obtain the well-known Boiling number, which takes into account the effect of heat flux, mass flux and latent heat on the heat transfer rate:

$$
\Psi_{9} \rightarrow \Pi_{10}^{-1} \cdot \Pi_{11}=\frac{q}{G i_{l v}}=B o
$$

Following to the procedure applied in the case of the pressure drop analysis, the power law notation combined with multiple regression analysis was used to correlate the two-phase Nusselt number and the unknown empirical coefficients were obtained by fitting the experimental data points based on the least square method. Again, any non-dimensional group which did not provide an improvement in accuracy in the prediction method was eliminated. As suggested by Kew and Cornwell (1997), the transition from macro-to-microscale for tubular geometries occurs at a Bond number Bd $=4$, being microscale when $\mathrm{Bd}<4$. Consequently, the prediction of the two-phase Nusselt number should be performed according to the value of the Bond number. When the experimental Bond number was less than 4 (microscale), the twophase Nusselt number was found to be associated to the homogeneous Weber number, Bond number, Boiling number, density ratio (liquid/vapor) and chevron angle as follows:

$$
N u_{t p}=982 \cdot \beta^{* 1.101} W e_{m}^{0.315} \mathrm{Bo}^{0.320} \rho^{*-0.224}
$$

Otherwise, for values of the Bond number greater than or equal to 4 (macroscale), the homogeneous Weber number was replaced by the regression technique by vapor Reynolds and liquid only Reynolds numbers. In this scenario, the twophase Nusselt number was correlated as:

$$
N u_{t p}=18.495 \cdot \beta^{* 0.248} \operatorname{Re}_{v}^{0.135} \operatorname{Re}_{l o}^{0.351} B d^{0.235} B o^{0.198} \rho^{* 0.223}
$$

According to these two expressions, the heat transfer in plate heat exchangers is enhanced by increasing chevron angle (much more so when $\mathrm{Bd}<4$ ). According to the two-phase flow theory in the channels, the heat transfer coefficient passing from macro-to-microscale should be enhanced because of the confinement effect, which provides a gradual expansion of the annular flow regime and the gradual suppression of the isolated bubble regime, and thus the transition appears earlier. This also promotes stronger turbulence in the flow coupled to the complex flow passage. The forced convection mechanism was found to be the dominant mechanism, even at low vapor quality. The Nusselt number rose with an increase of Boiling number due to the effect of the heat flux, which was important especially at the beginning of the plate when the flow initiates the evaporation process. Notably, the exponents on $\mathrm{q}$ in the two expressions are much less than 0.7, that common to nucleate pool boiling correlations improvised to match some test data as noted earlier. Moreover, the heat transfer rate increased with the Bond number and on the contrary, it decreased with the density ratio. Based on the proposed correlation, the behavior seemed to be the opposite and the reason may be due to the fact that the flow is primarily a complex film flow with little nucleate boiling over this entire range of corrugations. Finally, these two expressions can be considered as macro- and micro-scale plate heat exchanger flow boiling correlations.

A complete overview of the non-dimensions groups covered in the case of heat transfer is shown in Table 4 and Table 5, where the experimental range of each is reported for macroscale and microscale accordingly. 
Table 4: Range of dimensionless numbers for macroscale flow boiling heat transfer database.

\begin{tabular}{|c|c|c|}
\hline Parameter & Min value & Max value \\
\hline Nusselt number, $\mathrm{Nu}$ & $3.92 \cdot 10^{1}$ & $5.29 \cdot 10^{2}$ \\
\hline Homogeneous Weber number, $\mathrm{We}_{\mathrm{m}}$ & $2.41 \cdot 10^{-1}$ & $1.62 \cdot 10^{2}$ \\
\hline Liquid only Reynolds number, $\mathrm{Re}_{\mathrm{lo}}$ & $8.38 \cdot 10^{1}$ & $5.36 \cdot 10^{3}$ \\
\hline Vapor only Reynolds number, $\mathrm{Re}_{\mathrm{vo}}$ & $1.85 \cdot 10^{3}$ & $8.58 \cdot 10^{4}$ \\
\hline Density ratio, $\rho^{*}$ & $1.91 \cdot 10^{1}$ & $1.28 \cdot 10^{2}$ \\
\hline Reduced chevron angle, $\beta^{*}$ & $4.00 \cdot 10^{-1}$ & 1.00 \\
\hline Liquid Reynolds number, $\mathrm{Re}_{\mathrm{I}}$ & $3.48 \cdot 10^{1}$ & $5.32 \cdot 10^{3}$ \\
\hline Vapor Reynolds number, $\operatorname{Re}_{\mathrm{v}}$ & 7.94 & $3.45 \cdot 10^{4}$ \\
\hline Bond number, Bd & 4.33 & $7.89 \cdot 10^{1}$ \\
\hline Boiling number, Bo & $1.15 \cdot 10^{-4}$ & $3.75 \cdot 10^{-3}$ \\
\hline
\end{tabular}

Table 5: Range of dimensionless numbers for microscale flow boiling heat transfer database.

\begin{tabular}{lll}
\hline Parameter & Min value & Max value \\
\hline Nusselt number, Nu & 2.07 & $1.21 \cdot 10^{2}$ \\
Homogeneous Weber number, We & & $4.15 \cdot 10^{1}$ \\
Liquid only Reynolds number, Relo & $2.67 \cdot 10^{-2}$ & $2.72 \cdot 10^{3}$ \\
Vapor only Reynolds number, Re ${ }_{\mathrm{v}}$ & $4.12 \cdot 10^{1}$ & $4.22 \cdot 10^{4}$ \\
Density ratio, $\rho^{*}$ & $1.58 \cdot 10^{3}$ & $1.35 \cdot 10^{3}$ \\
Reduced chevron angle, $\beta^{*}$ & $7.75 \cdot 10^{1}$ & $9.29 \cdot 10^{-1}$ \\
Liquid Reynolds number, Rel & $4.29 \cdot 10^{-1}$ & $2.58 \cdot 10^{3}$ \\
Vapor Reynolds number, Re & $2.34 \cdot 10^{1}$ & $6.52 \cdot 10^{3}$ \\
Bond number, Bd & 8.58 & 3.76 \\
Boiling number, Bo & 1.89 & $4.05 \cdot 10^{-3}$ \\
\hline
\end{tabular}

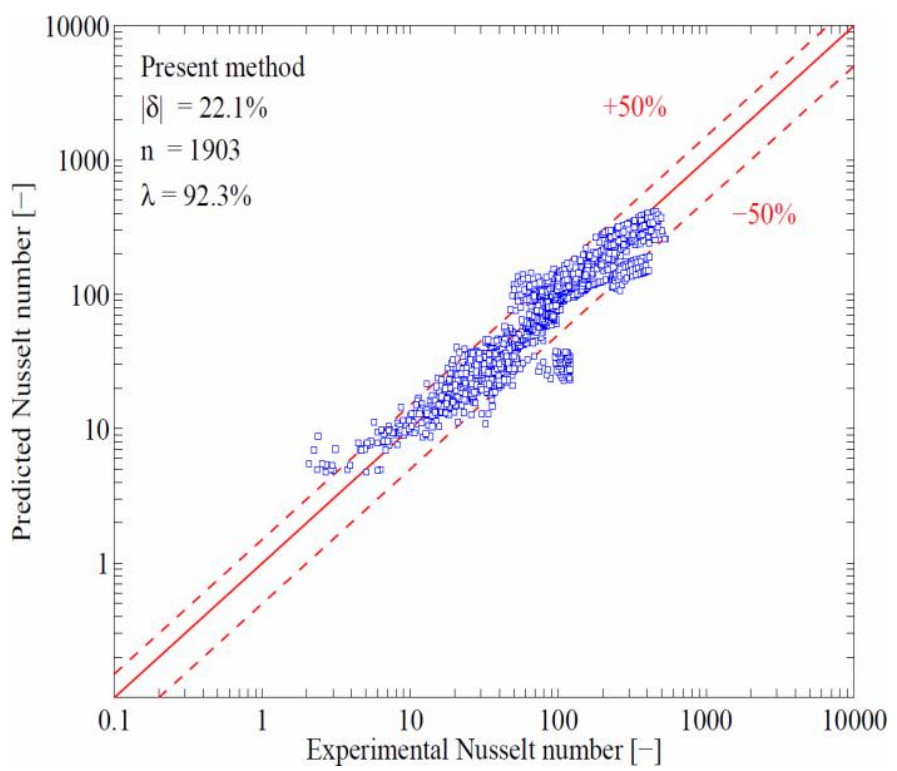

Fig. 9: Flow boiling prediction methods for heat transfer within PHEs: predicted against experimental Nusselt number.

Note that for the frictional pressure drop and heat transfer calculation, the hydraulic diameter is only used as the characteristic dimension of the channels. The mass flux is that calculated using the actual cross-sectional area of axial flow along the plate, not using the cross-sectional area that could be calculated using the hydraulic diameter. 
Furthermore, the actual cross-sectional area is calculated as $\mathrm{b} \cdot \mathrm{W}$ and the hydraulic diameter for the plate is calculated as $2 \mathrm{~b} / \varphi$.

In Fig. 9 the newly proposed methods are shown compared to the entire database. It can be concluded that there is a good agreement between the predicted and experimental data, since the prediction methods predicted $92.3 \%$ of the entire flow boiling databank (which included 1903 data points) with a mean absolute error of $22.1 \%$ within $\pm 50 \%$ while $76.1 \%$ could be captured within $\pm 30 \%$. Compared to Fig. 6 , the improvement is visibly evident.

Table 6: Two-phase heat transfer and frictional pressure drop predicted by the correlations along the columns compared to the entire database.

\begin{tabular}{|c|c|c|c|c|c|c|c|c|c|}
\hline \multirow[t]{2}{*}{ Author(s) } & \multicolumn{4}{|c|}{$\begin{array}{l}\text { Heat transfer database (1903 data) } \\
\text { [Table 1, Part 1] }\end{array}$} & \multirow[t]{2}{*}{ Author(s) } & \multicolumn{4}{|c|}{$\begin{array}{l}\text { Pressure drop database (1513 data) } \\
\text { [Table 1, Part 1] }\end{array}$} \\
\hline & $|\delta| \%$ & $\delta \%$ & $\xi \%$ & $\lambda \%$ & & $|\delta| \%$ & $\delta \%$ & $\xi \%$ & $\lambda \%$ \\
\hline $\begin{array}{l}\text { Danilova et al. } \\
\text { (1981) }\end{array}$ & 28.3 & 0.97 & 66.4 & 88.2 & $\begin{array}{l}\text { Yan and Lin } \\
\text { (1999) }\end{array}$ & $\begin{array}{l}2427 . \\
1\end{array}$ & 2407.3 & 12.9 & 20.4 \\
\hline $\begin{array}{l}\text { Yan and Lin } \\
\text { (1999) }\end{array}$ & 79 & -78.6 & 1.8 & 4.5 & $\begin{array}{l}\text { Hsieh and Lin } \\
(2002 b)\end{array}$ & $\begin{array}{l}1032 . \\
6\end{array}$ & 1032.6 & 18.6 & 26.1 \\
\hline $\begin{array}{l}\text { Hsieh and Lin } \\
(2002 b)\end{array}$ & 44.7 & 30.7 & 44.7 & 67.4 & $\begin{array}{l}\text { Hsieh and Lin } \\
(2003 c)\end{array}$ & 846 & 836.8 & 19.7 & 29.5 \\
\hline $\begin{array}{l}\text { Hsieh and Lin } \\
(2003 c)\end{array}$ & 52.1 & 44.3 & 51.7 & 69 & $\begin{array}{l}\text { Sterner and Sunden } \\
(2006 \mathrm{c})\end{array}$ & 225 & 184.9 & 13.8 & 22.7 \\
\hline $\begin{array}{l}\text { Sterner and Sunden } \\
(2006 \mathrm{c})\end{array}$ & 1238.6 & 1205.4 & 9.1 & 16.3 & $\begin{array}{l}\text { Han et al. } \\
(2003 b)\end{array}$ & 292 & 256.1 & 19 & 31.5 \\
\hline $\begin{array}{l}\text { Downoski and } \\
\text { Kandlikar (2000a) }\end{array}$ & 47.6 & 30 & 54.1 & 71.3 & $\begin{array}{l}\text { Ayub } \\
(2003 a)\end{array}$ & 68.8 & -17.8 & 23 & 41.5 \\
\hline $\begin{array}{l}\text { Palmer et al. } \\
(2000 b)\end{array}$ & 108.1 & 87 & 40.4 & 61.7 & $\begin{array}{l}\text { Nilpueng and } \\
\text { Wongwises } \\
\text { (2010a) }\end{array}$ & 72.5 & -72.5 & 2.7 & 10.2 \\
\hline $\begin{array}{l}\text { Han et al. } \\
\text { (2003b) }\end{array}$ & 32.9 & -15.3 & 47.0 & 89 & $\begin{array}{l}\text { Jokar et al. } \\
(2006 a)\end{array}$ & 812.2 & 764.5 & 14.3 & 21.6 \\
\hline $\begin{array}{l}\text { Ayub } \\
(2003 a)\end{array}$ & 80.1 & 22.1 & 31.7 & 53.6 & $\begin{array}{l}\text { Khan et al. } \\
(2014 b, 2012 b, 2012 c)\end{array}$ & 37.8 & -8.7 & 57.6 & 76.1 \\
\hline $\begin{array}{l}\text { Palm and Claesson } \\
\text { (2006b) }\end{array}$ & 177.9 & 177.3 & 8.6 & 15.8 & $\begin{array}{l}\text { Taboas et al. } \\
(2012 f)\end{array}$ & 51.7 & -32.6 & 26.4 & 46.1 \\
\hline $\begin{array}{l}\text { Jokar et al. } \\
\text { (2006a) }\end{array}$ & 70.1 & 15 & 25.4 & 49.1 & $\begin{array}{l}\text { Huang et al. } \\
(2012 a)\end{array}$ & 173 & 150.8 & 25.3 & 40.8 \\
\hline $\begin{array}{l}\text { Khan et al. } \\
\text { (2014b, 2012b, 2012c) }\end{array}$ & 120.8 & 48.6 & 17.1 & 31.2 & $\begin{array}{l}\text { Park and Kim } \\
\text { (2004) }\end{array}$ & 6960 & 6960 & 7.5 & 17.9 \\
\hline $\begin{array}{l}\text { Taboas et al. } \\
(2012 \mathrm{f})\end{array}$ & 48.1 & -16 & 25.8 & 62.3 & $\begin{array}{l}\text { Lee et al. } \\
(2014 a)\end{array}$ & 83.9 & -83.9 & 0.9 & 2.1 \\
\hline $\begin{array}{l}\text { Huang et al. } \\
\text { (2012a) }\end{array}$ & 34.8 & -8.6 & 45.1 & 79.5 & Present method & 21.5 & -6.8 & 74.1 & 90.9 \\
\hline $\begin{array}{l}\text { Park and Kim } \\
\text { (2004) }\end{array}$ & 268.2 & 265.2 & 27.9 & 31.1 & & & & & \\
\hline $\begin{array}{l}\text { Lee et al. } \\
(2014 a)\end{array}$ & 351.2 & 310.4 & 16.3 & 19.5 & & & & & \\
\hline Present method & 22.1 & -5.8 & 76.1 & 92.3 & & & & & \\
\hline
\end{tabular}


Table 6 illustrates the comparison between all the prediction methods available in the open literature for heat transfer coefficient and frictional pressure gradient against the entire set of experimental data points which have been selected to build the present flow boiling database. It can be observed that only two models were able to predict with a discrete agreement the experimental flow boiling heat transfer: the Danilova et al. (1981) method (mean absolute error of $28.3 \%$ and $88.2 \%$ of the data in the range $\pm 50 \%$ ) and Han et al. (2003b) method (mean absolute error of $32.9 \%$ and $89 \%$ of the data in the range $\pm 50 \%$ ). Within the narrower range of $\pm 30 \%$ required for heat exchanger design, these two methods are notably less effective than the present method, $66.4 \%$ and $47 \%$ versus $76.1 \%$.

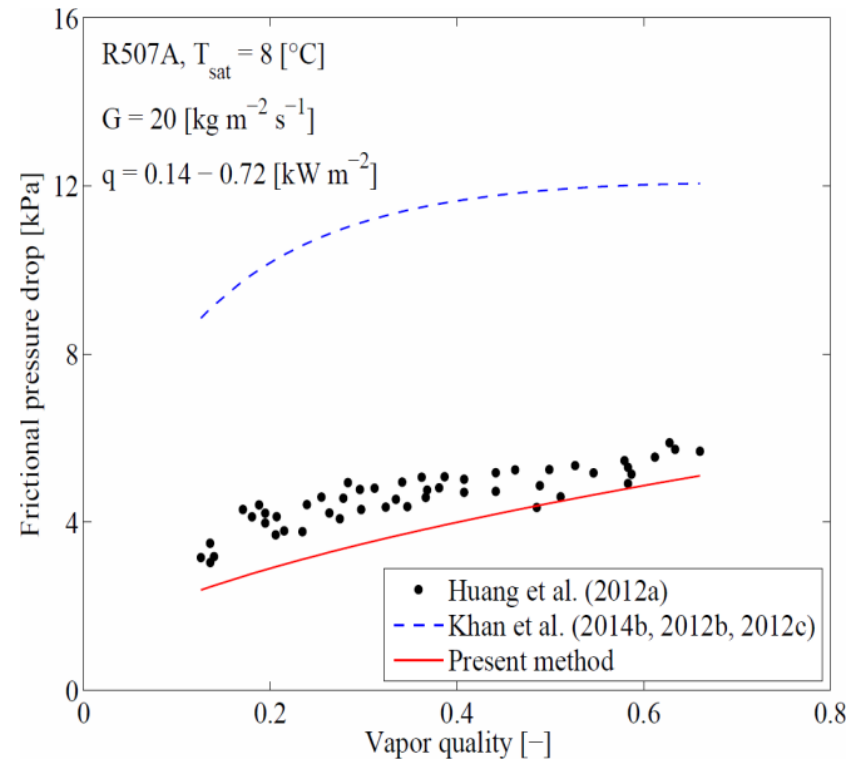

(a)

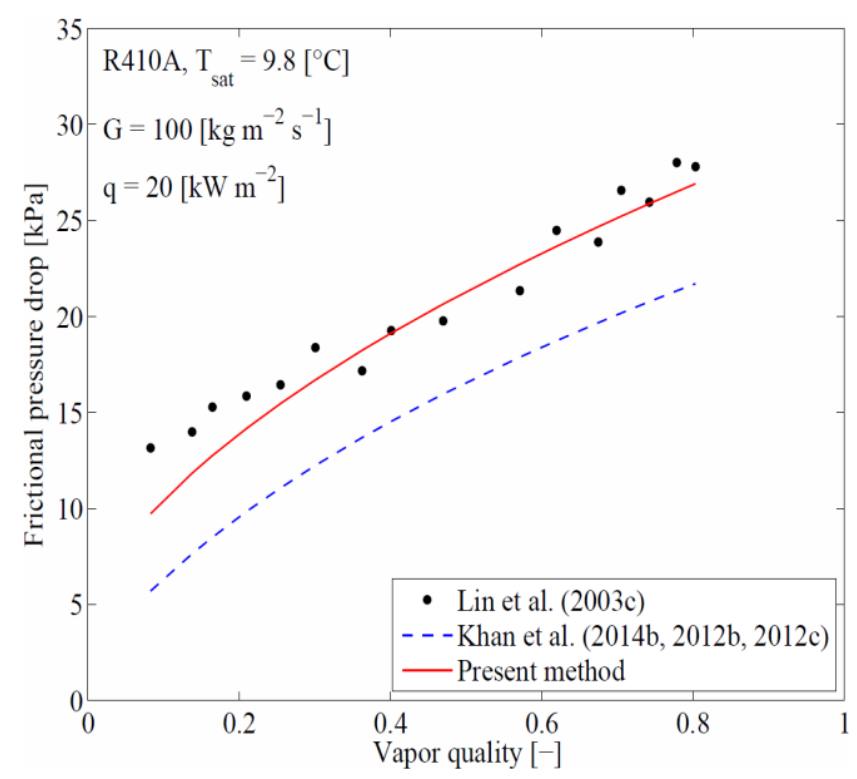

(c)

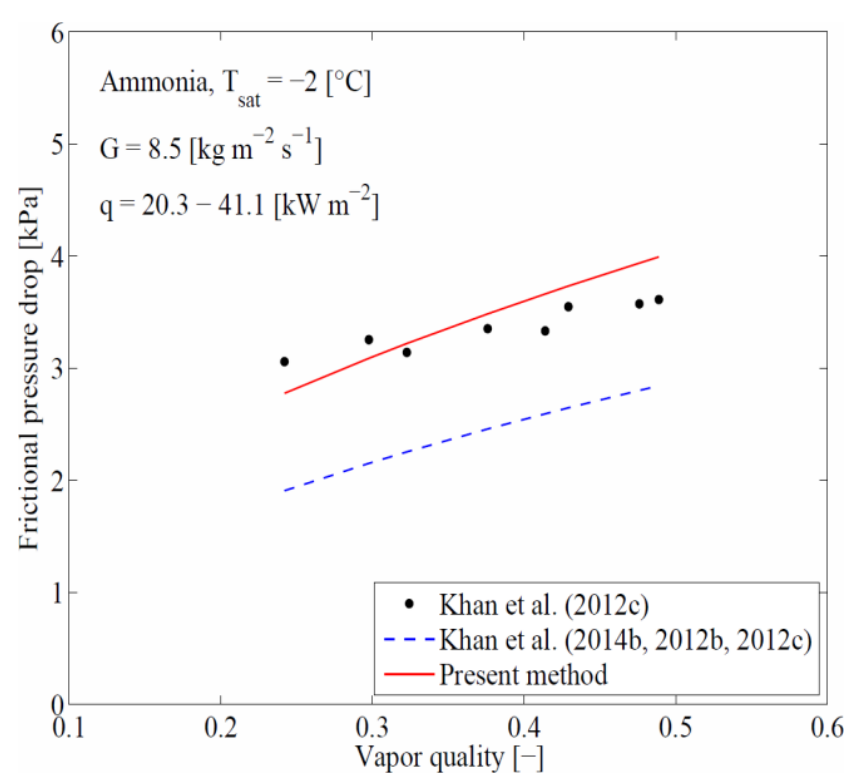

(b)

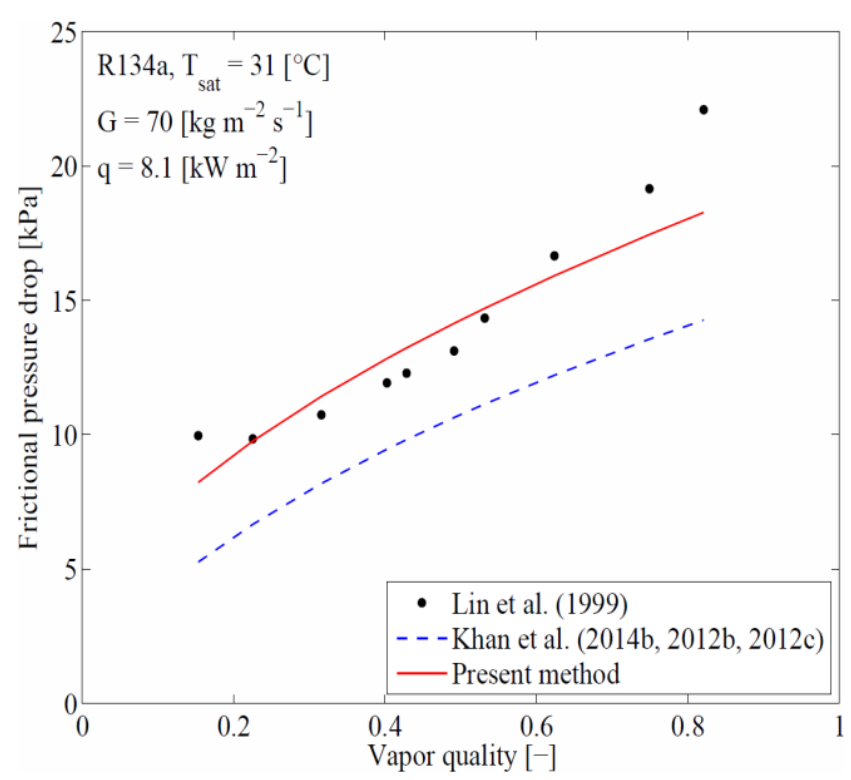

(d)

Fig. 10: Comparison between the predicted frictional pressure drops by Khan et al. (2014b, 2012b, 2012c) and new method plotted versus vapor quality versus: (a) Huang et al. data (2012a), (b) Khan et al. data (2014b, 2012b, 2012c), (c) and (d) Lin et al. data (2002a, 2003c, 2002b, 1999).

In the case of two-phase pressure drops, only the Khan et al. (2014b, 2012b, 2012c) correlation could be used to predict the hydraulic performance within plate heat exchangers (mean absolute error of $37.8 \%$ and $76.1 \%$ of the data in the range $\pm 50 \%$ ), while the other correlations largely underestimate or overestimate the flow boiling pressure drop database. Compared to the above methods, the new method here can predict both flow boiling heat transfer with a mean 
absolute error of $22.1 \%$ and $92.3 \%$ of the data estimated in the range $\pm 50 \%$ and pressure drop database with a mean absolute error of $21.5 \%$ and $90.9 \%$ predicted data within $\pm 50 \%$.

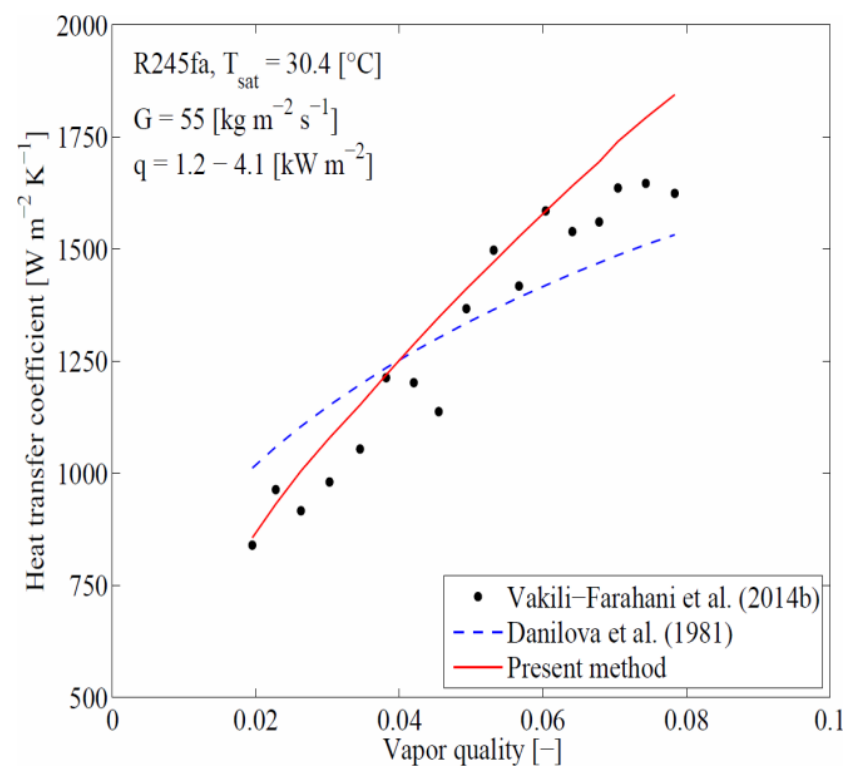

(a)

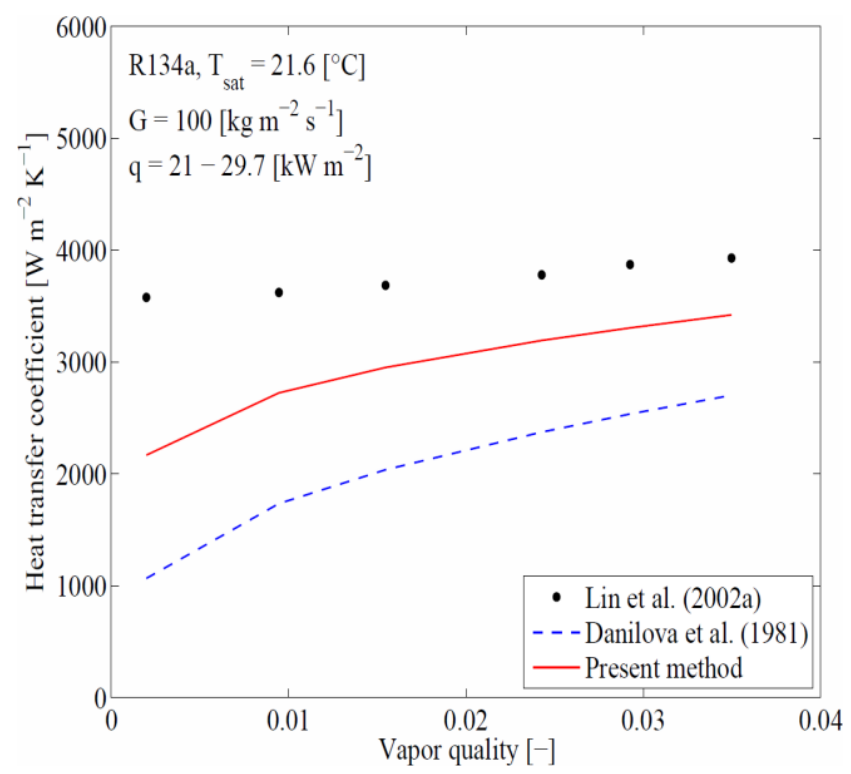

(c)

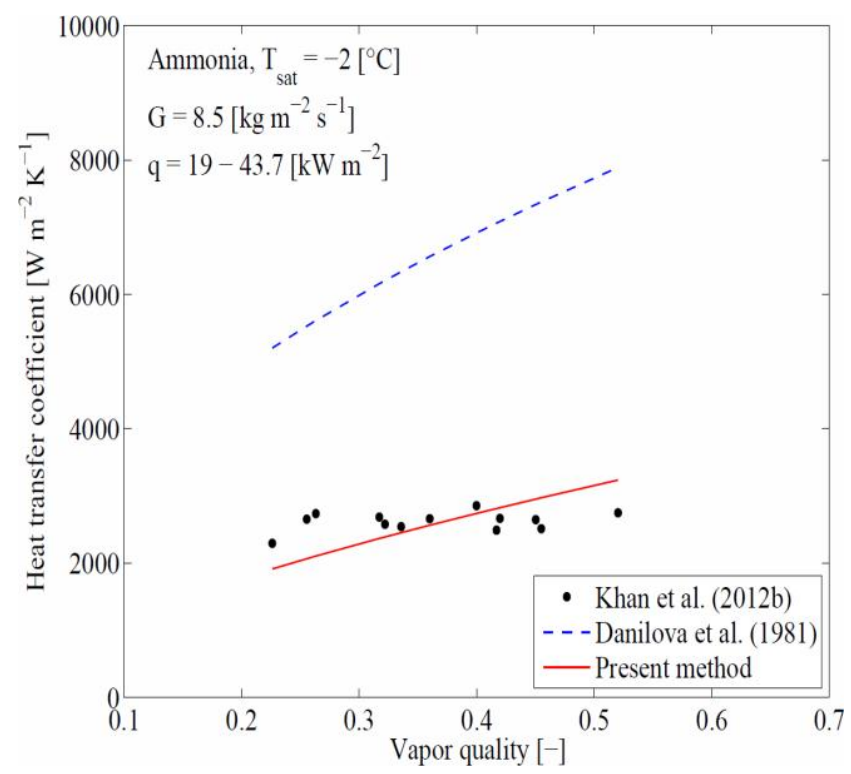

(b)

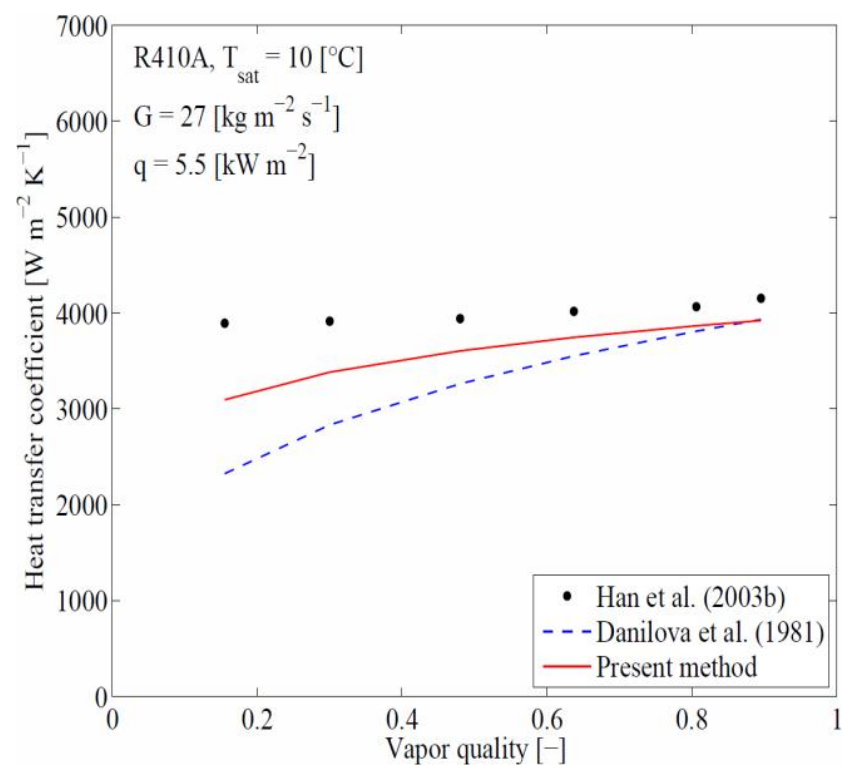

(d)

Fig. 11: Comparison between the experimental and the predicted heat transfer coefficients predicted by Danilova et al. (1981) and new method, plotted versus vapor quality: (a) Vakili-Farahani et al. data (2014c, 2014d), (b) Khan et al. data (2014b, 2012b, 2012c), (c) Lin et al. data (2002a, 2003c, 2002b, 1999), (d) Han et al. data (2003b).

To highlight the predicted capabilities of the new frictional pressure drop method versus the next best methods, several more comparisons have been performed. One can see the new method follow the trends in the data and it works for fluids as diverse as R507A and ammonia. As can be seen from the Fig. 10, the new model closely predicted the four independent databases selected for this comparison. In the Fig. 10b, the proposed model exhibited better capability to evaluate the hydraulic performance of a $60^{\circ}$ chevron plate with ammonia compared to their own correlation, which slightly underestimated their experimental data. Furthermore, it has to be pointed out that only in the case of the Lin et al. (2002a, 2003c, 2002b, 1999) data at relatively high mass flux, the new method was not able to follow the entire trend. At low vapor quality the frictional pressure drop was underestimated as well as in the turbulent flow region, in which the 
Fanning friction factor approximated to an asymptote and the frictional pressure drop was strongly dependent to the mean vapor quality. In conclusion, the proposed model underestimated the pressure drop database provide by Lee et al. (2014a) since flow boiling of water at low mass fluxes exhibits different behavior compared to the traditional refrigerants (see Table 2).

Fig. 11 depicts a similar comparison, but now the heat transfer coefficient against the vapor quality was examined. It can be observed that the new method predicts the heat transfer rate with a good agreement to the experimental data points for both microscale (Vakili-Farahani et al (2014c, 2014d) and Khan et al. data (2014b, 2012b, 2012c)) and macroscale (Lin et al. (2002a, 2003c, 2002b, 1999) and Han et al. data (2003b)) conditions as well as different corrugation angles. In particular, in the case of ammonia, the prediction method provided by Danilova et al. (1981) strongly overestimated the experimental values; thus, their extrapolation at low mass fluxes and $30^{\circ}$ chevron angle plate with ammonia is not recommended. It has also to be highlighted that the present method could only predict qualitatively the subcooled flow boiling data of the Lin group's due to the fact that no subcooling parameter was introduced into the prediction method. Moreover, the present prediction method underestimated the database collected by Lee et al. (2014a) because no parameter was involved to take into account the heat transfer within the dry-out region, thus a future study should look specifically at these conditions. It has also to be highlighted, that the new correlation underestimated the databank provided by Park and Kim (2004) and the reason can be due to the fact that the authors investigated flow boiling of refrigerants inside an oblong shell and plate heat exchanger (see Table 1).

\section{Conclusions}

In the current work, the most important outcomes regarding the prediction of the thermal and hydraulic performances of PHE's can be summarized as follows:

- The sensitivity analysis illustrated the effects of the main geometrical parameters on the performance of the plate heat exchangers. The heat transfer coefficient and the associated frictional pressure drop rise with an increase of chevron angle, while on the contrary they decrease when the corrugation pitch and hydraulic diameter are increased.

- The leading published methods were compared statistically versus large multi-lab databases for flow boiling heat transfer coefficients and frictional pressure drops in PHE's. The flow boiling heat transfer rates were discretely predicted by the Danilova et al. (1981) and Han et al. (2003b) methods, while in the case of frictional pressure drops the best existing method was that of Khan et al. (2014b, 2012b, 2012c). Also, it was shown that most of the correlations should not be extrapolated out of their original range of test conditions.

- Based on a dimensional and multiple regression analysis, new prediction methods for flow boiling heat transfer and frictional pressure drop within plate heat exchangers have been proposed. These methods were developed from 1903 heat transfer and 1513 frictional pressure drop data points, respectively, and were proved to work better than any other published method over a very wide range of operating conditions, plate designs and fluids (including ammonia).

\section{Acknowledgement}

The authors are thankful for the support of the current work: ONR Office of Naval Research Science \& Technology and LTCM heat and mass transfer laboratory. The authors wish to thank U.S. Office of Naval Research (in particular the heat transfer program director, Mark Spector, in the Ship Systems and Engineering Division) for the financial support provided for the project N000141210398 for the PhD thesis of R.L. Amalfi. 


\section{References}

Ayub, Z.H., 2003a. Plate Heat Exchanger Literature Survey and New Heat Transfer and Pressure Drop Correlations for Refrigerant Evaporators. Heat Transfer Eng. 24, 3-16.

Cioncolini, A., Thome, J.R., 2009. Unified Macro-to-Microscale Method to Predict Two-Phase Frictional Pressure Drops of Annular Flows. Int. J. Multiphase Flow. 35, 1138-1148.

Cooper, M.G., 1984. Heat Flow Rates in Saturated Nucleate Pool Boiling-a Wide-Ranging Examination Using Reduced Properties. Advances in Heat Transfer. 16, 157-239.

Danilova, G.N., Azarskov, V.M., Zemskov, B.B., 1981. Teploobmen V Plastinchatihispariteljan Razichnole Geometri Heat Transfer in Plate Evaporators of Different Geometry. Kholod. Tek. 4, 25-31.

Djordjevic, E., Kabelac, S., 2008. Flow Boiling of R134a and Ammonia in a Plate Heat Exchanger. Int. J. Heat Mass Trans. 51, 6235-6242.

Donowski, V.D., Kandlikar, S.G., 2000a. Correlating Evaporation Heat Transfer Coefficient of Refrigerant R-134a in a Plate Heat Exchanger. Engineering Foundation Conference on Pool and Flow Boiling, Alaska, Paper 154.

Eungchan Lee, H.K., Yongchan Kim, 2014a. Flow Boiling Heat Transfer and Pressure Drop of Water in a Plate Heat Exchanger with Corrugated Channels at Low Mass Flux Conditions. Int. J. Heat Mass Transfer. 77, 37-45.

Han, D.H., Lee, K.J., Kim, Y.H., 2003b. Experiments on the Characteristics of Evaporation of R410a in Brazed Plate Heat Exchangers with Different Geometric Configurations. Applied Thermal Eng. 23, 1209-1225.

Hsieh, Y.Y., Chiang, L.J., Lin, T.F., 2002a. Subcooled Flow Boiling Heat Transfer of R134a and the Associated Bubble Characteristics in a Vertical Plate Heat Exchanger. Int. J. Heat Mass Transfer. 45, 1791-1806.

Hsieh, Y.Y., Lin, T.F., 2003c. Evaporation Heat Transfer and Pressure Drop of Refrigerant R410a Flow in a Vertical Plate Heat Exchanger. J. Heat Transfer. 125, 852-857.

Hsieh, Y.Y., Lin, T.F., 2002b. Saturated Flow Boiling Heat Transfer and Pressure Drop of Refrigerant R-410a in a Vertical Plate Heat Exchanger. Int. J. Heat Mass Transfer. 45, 1033-1044.

Huang, J., Sheer, T., Bailey-McEwan, M., 2012a. Heat Transfer and Pressure Drop in Plate Heat Exchanger Refrigerant Evaporators. Int. J. Refrigeration. 35, 325-335.

Jokar, A., Hosni, M.H., Eckels, S.J., 2006a. Dimensional Analysis on the Evaporation and Condensation of Refrigerant R-134a in Minichannel Plate Heat Exchangers. Applied Thermal Eng. 26, 2287-2300.

Kew, P.A., Cornwell, K., 1997. Correlations for the Prediction of Boiling Heat Transfer in Small-Diameter Channels. Appl. Therm. Eng. 17, 705-715.

Khan, M.S., Khan, T.S., Chyu, M.C., Ayub, Z.H., 2014b. Evaporation Heat Transfer and Pressure Drop of Ammonia in a Mixed Configuration Chevron Plate Heat Exchanger. Int. J. Refrigeration. 41, 92-102.

Khan, M.S., Khan, T.S., Chyu, M.C., Ayub, Z.H., 2012b. Experimental Investigation of Evaporation Heat Transfer and Pressure Drop of Ammonia in a $30^{\circ}$ Chevron Plate Heat Exchanger. Int. J. Refrigeration. 35, 1757-1765.

Khan, T.S., Khan, M.S., Chyu, M.C., Ayub, Z.H., 2012c. Experimental Investigation of Evaporation Heat Transfer and Pressure Drop of Ammonia in a $60^{\circ}$ Chevron Plate Heat Exchanger. Int. J. Refrigeration. 35, 336-348.

Longo, G.A., 2012d. Hydrocarbon Refrigerant Vaporization inside a Brazed Plate Heat Exchanger. J. Heat Transfer. 134, 101801_101801-101810.

Longo, G.A., 2012e. Vaporisation of the Low Gwp Refrigerant Hfo1234yf inside a Brazed Plate Heat Exchanger. Int. J. Refrigeration. 35, 952-961.

Longo, G.A., Gasparella, A., 2007a. Heat Transfer and Pressure Drop During Hfc Refrigerant Vaporisation inside a Brazed Plate Heat Exchanger. Int. J. Heat Mass Trans. 50, 5194-5203.

Longo, G.A., Gasparella, A., 2007b. Hfc-410a Vaporisation inside a Commercial Brazed Plate Heat Exchanger. Exp. Therm. Fluid Sci. 32, 107-116.

Longo, G.A., Gasparella, A., 2007c. Refrigerant R134a Vaporisation Heat Transfer and Pressure Drop inside a Small Brazed Plate Heat Exchanger. Int. J. Refrigeration. 30, 821-830. 
Nilpueng, K., Wongwises, S., 2010a. Two-Phase Gas-Liquid Flow Characteristics inside a Plate Heat Exchanger. Exp. Therm. Fluid Sci. 34, 1217-1229.

Ouazia, B., 2001a. Evaporation Heat Transfer and Pressure Drop of Hfc-R134a inside a Plate Heat Exchanger. ASME Int. Mech. Eng. Congress and Exposition, IMECE2001/PID-25613, New York, NY, 115-123.

Palm, B., Claesson, J., 2006b. Plate Heat Exchangers: Calculation Methods for Singleand Two-Phase Flow. Heat Transfer Eng. 27, 88-98.

Palmer, S.C., Vance Payne, W., Domanski, P.A., 2000b. Evaporation and Condensation Heat Transfer Performance of Flammable Refrigerants in a Brazed Plate Heat Exchanger.

Park, J.H., Kim, Y.S., 2004. Evaporation Heat Transfer and Pressure Drop Characteristics of R134a in the Oblong Shell and Plate Heat Exchanger. KSME Int. J. 18, 2284-2293.

Shah, R.K., Focke, W.W., 1988. Plate Heat Exchangers and Their Design Theory. Heat Transfer Equipment Design, Hemisphere Publishing, Washington, DC, 227-254.

Sterner, D., Sundén, B., 2006c. Performance of Plate Heat Exchangers for Evaporation of Ammonia. Heat Transfer Eng. $27,45-55$.

Táboas, F., Vallès, M., Bourouis, M., Coronas, A., 2012f. Assessment of Boiling Heat Transfer and Pressure Drop Correlations of Ammonia/Water Mixture in a Plate Heat Exchanger. Int. J. Refrigeration. 35, 633-644.

Táboas, F., Vallès, M., Bourouis, M., Coronas, A., 2010b. Flow Boiling Heat Transfer of Ammonia/Water Mixture in a Plate Heat Exchanger. Int. J. Refrigeration. 33, 695-705.

Tribbe, C., Müller-Steinhagen, H.M., 2001b. Gas/Liquid Flow in Plate-and-Frame Heat Exchangers - Part 1: Pressure Drop Measurements. Heat Transfer Eng. 22, 5-11.

Tribbe, C., Müller-Steinhagen, H.M., 2001c. Gas/Liquid Flow in Plate-and-Frame Heat Exchangers - Part 2: Two-Phase Multiplier and Flow Pattern Analysis. Heat Transfer Eng. 22, 12-21.

Vakili-Farahani, F., Amalfi, R.L., Thome, J.R., 2014c. Two-Phase Flow and Boiling of R245fa in a 1mm Pressing Depth Plate Heat Exchanger - Part 1: Adiabatic Pressure Drop. Int. J. Interfacial Phenomena and Heat Transfer. 2, 325 342.

Vakili-Farahani, F., Amalfi, R.L., Thome, J.R., 2014d. Two-Phase Flow and Boiling of R245fa in a 1mm Pressing Depth Plate Heat Exchanger - Part 2: Flow Boiling Heat Transfer. Int. J. Interfacial Phenomena and Heat Transfer. 2 , 343-361.

Yan, Y.Y., Lin, T.F., 1999. Evaporation Heat Transfer and Pressure Drop of Refrigerant R134a in a Plate Heat Exchanger. ASME J. Heat Transfer. 121, 118-127. 